\title{
Growth, physiological, nutrient uptake efficiency and shade tolerance responses of cacao genotypes under different shades
}

Enrique Arévalo-Gardini ${ }^{\mathrm{a}, \mathrm{b}^{*}}$, Abel Farfán ${ }^{\mathrm{a}}$, Fiorella Barraza ${ }^{\mathrm{a}, \mathrm{c}}$, César O. Arévalo-Hernández ${ }^{\mathrm{a}}$, Luis B. Zuñiga ${ }^{a}$, Julio Alegred, Virupax C. Baligare

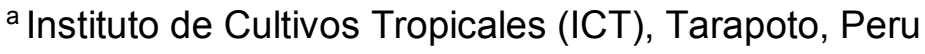

b Universidad Nacional Autonoma de Alto Amazonas (UNAAA), Yurimaguas, Peru

${ }^{c}$ Department of Renewable Resources, University of Alberta, Edmonton, AB, T6G 2G7, Canada

' Universidad Nacional Agraria La Molina (UNALM), Lima, Peru

e U.S. Department of Agriculture/Agricultural Research Service, Beltsville Agricultural Research Center, Beltsville, MD 20705, USA

*corresponding author: enriquearevaloga@gmail.com

\section{Highlights:}

- Significant interactions observed between shade and cacao genotypes

- Nutrient use efficiency increased with reduced shade

- Fourteen cacao genotypes were tolerant to high shade

- Intraspecific difference observed for growth, physiology and nutritional traits at different shade levels

Keywords: Theobroma cacao, light, abiotic stress, physiology, plant nutrition

\begin{abstract}
Cacao is an understory plant cultivated under full-sun monocultures to multi-strata agroforestry systems, where cocoa trees are planted together with fruit, timber, firewood, and leguminous trees, or grown within thinned native forests. Under agroforestry systems of cultivation cacao is subjected to excess shade due to high density of shade trees, and over grown or unmanaged pruning of shade trees. Cacao is tolerant to shade, and maximum photosynthetic rate occurs around irradiance of $400 \mu \mathrm{mol} \mathrm{m}^{-2} \mathrm{~s}^{-1}$ but excess shade reduces the irradiance further which is detrimental to photosynthesis and growth functions. Intra specific variation is known to exist in cacao for the required saturation irradiance. A greenhouse study was implemented with 58 cacao genotypes selected from four geographically diverse groups: (i) wild cacao from river basins of the Peruvian
\end{abstract}


Amazon, (PWC), (ii) Peruvian farmers' collection (PFC), (iii) Brazilian cacao collection (BCC) and (iv) national and international cacao collections (NIC). All the cacao genotypes were subjected to $50 \%$ and $80 \%$ shade where photosynthetic photon flux density (PPFD) was 1000 and $400 \mu \mathrm{mol} \mathrm{m} \mathrm{m}^{-2} \mathrm{~s}^{-1}$ respectively. Intra specific variations were observed for growth, physiological and nutritional traits and tolerance to shade. Cacao genotypes tolerant to shade were: UNG-77 and UGU-130 from PWC; ICT-2173, ICT-2142, ICT2172, ICT-1506, ICT-1087, and ICT-2171 from the PFC; PH-21, CA-14, PH-990 and PH144 from BCC; and ICS-1, ICS-39, UF-613 and POUND-12 from NIC. Genotypes that tolerate excess shade might be useful plant types to maintain productivity and sustainability in agroforestry systems of cacao management.

\section{Introduction}

Beyond the basic concept of shade tolerance, which is defined as the minimum light required for a plant to survive and develop in different strata, this factor involves a wide range of effects that are connected with several aspects of the plant life cycle as well as with ecosystem dynamics [1-4]. Adaptation of species to shade is still poorly understood [5], and the positive or negative effects are sometimes contradictory, which is the case of cacao [6].

Cacao (Theobroma cacao) is an understory plant $[7,8]$ cultivated under different cropping systems: from full-sun monocultures to multi-strata agroforestry systems, where cocoa trees are planted together with fruit, timber, firewood, and leguminous trees, or within thinned forests $[7,8]$. The use of shade trees is a common agricultural and sustainable practice in cacao production systems [9-16]. These trees act as a protection barrier against stressful environmental conditions such as extreme temperature, solar radiation, drought, and intense rainfall and wind $[6,7,17,18]$.

Several benefits have been attributed to cacao growing within shaded agroforestry systems such as control of diseases or insect attacks, maintaining soil fertility, enhancing nutrient cycling, reducing soil erosion and deforestation, increasing tree diversity, mitigating climatic changes thorough $C$ sequestration, helping to reduce the use of pesticides and fertilizer applications [13, 19-26].

However, some authors report that lower yields have been observed in shaded cacao systems, mostly related to climate conditions (i.e. precipitation and temperature) [27-30]. Even so, farmers can get more profitable net revenues because of the lower maintenance cost (compare to conventional systems) and higher prices obtained per kilogram of cocoa beans [31].

In other situations, cacao yield is affected due to the presence of too many trees, trees with large canopies and poorly managed tree canopy structure [24,32]. Under such 
conditions the longevity of the plantation could also be affected [33], therefore it is advantageous to adopt cacao genotypes that maintain their productivity even under reduced irradiance.

A definition of an optimum shade level for cocoa is needed $[7,17,33]$ as well as a selection of the appropriate shade species to avoid the detrimental effects of shade [6]. It is also important to mention that the need of shade may not be required in all cacao-growing regions especially in island and heavy cloud cover ecosystems [6].

In tropical forests, understory plants receive a photosynthetic photon flux density (PPFD) of between 5 and $25 \mu \mathrm{mol} \mathrm{m} \mathrm{m}^{-2} \mathrm{~s}^{-1}$ or 1 to $2 \%$ of the irradiance obtained above the tree canopy level but they also intermittently get high levels of PPFD in the form of sunflecks [34-40]. Miyaji et al. [41] reported that the light intensity (at full daylight) above the cacao canopy shaded by trees in Bahia, Brazil was between 30 and $100 \%$ and between 4 and $10 \%$ at ground level. In another study conducted in Alto Beni (Bolivia); Niether et al. [18], measured the light levels of various cacao systems and determined a PPFD between 1580 and $2028 \mu \mathrm{mol} \mathrm{m} \mathrm{m}^{-2} \mathrm{~s}^{-1}$ above the canopy. In contrast, in an open sun monoculture, irradiance varied between 985 and $1546 \mu \mathrm{mol} \mathrm{m} \mathrm{m}^{-2} \mathrm{~s}^{-1}$ depending on canopy pruning (before and after, respectively). Consequently, light levels have significant influence on growth, physiological traits and nutrition of cacao [33,42-44].

It has been reported that maximum photosynthesis in cacao occurs at a PPFD of 350 to $550 \mu \mathrm{mol} \mathrm{m} \mathrm{m}^{-2} \mathrm{~s}^{-1}$, which is about 20 to $25 \%$ of the intensity of full sunlight [42, 45-47]. In some cacao genotype, an increase of PPFD from 50 to $400 \mu \mathrm{mol} \mathrm{m}{ }^{-2} \mathrm{~s}^{-1}$ entailed an increase of the net photosynthetic rate $\left(P_{N}\right)$ by about $50 \%$, but further increases (up to $1500 \mu \mathrm{mol} \mathrm{m} \mathrm{m}^{-2} \mathrm{~s}^{-1}$ ) had no effect, indicating that very little radiant energy is required to support efficient $P_{\mathrm{N}}$ in cacao [43].

Variations in morphological characteristics among different genotypes have been reported in cacao $[48,49]$. At the same time, these characteristics have been influenced by the level of irradiance [10, 11, 43, 50]. Baligar et al. [43] reported that in juvenile cacao genotypes increasing PPFD from 65 to $190 \mu \mathrm{mol} \mathrm{m}{ }^{-2} \mathrm{~s}^{-1}$ increased shoot and root growth, mineral nutrition and net assimilation rate. Thus, identification of plant traits for growth and physiological parameters that are influenced by light quality and intensity (PPFD) will help to identify cacao genotypes that could perform well under a range of light intensities (or shade levels).

The aim of this research was to evaluate the effects of shade (PPFD, Photosynthetic Photon Flux Density) on growth, physiological, macronutrients and micronutrients use efficiency and shade tolerance responses of national (wild, domesticated) and international cacao genotypes. 


\section{Material and methods}

\subsection{Experimental conditions}

\subsubsection{Cacao genotypes}

The greenhouse experiment was implemented at the Instituto de Cultivos Tropicales-ICT (Tropical Crops Institute), Tarapoto, San Martin, Peru. A total of 58 cacao genotypes from the germplasm bank located at "El Choclino" experimental station was selected from four geographic origins: (i) wild cacao from river basins of Peruvian Amazon collection (PWC), (ii) Peruvian farmers' collection (PFC), (iii). Brazilian cacao collection (BCC) and (iv). National and International cacao collection (NIC). Several expeditions were under taken by ICT during 2008 to collect wild cacao genotypes from the river basins of Aypena (AYP), Pastaza (PAS), Ungumayo (UGU) and Ungurahui rivers (UNG); on the other hand, Peruvian farmers' collections were made by ICT during 2002 to 2004 in the Provinces of Mariscal Cáceres and Tocache of San Martin department at North-East, Peru. Brazilian clones were from CEPLAC/CEPEC (Comissao Executiva do Plano da Lavoura Cacaueira/Centro de Pesquisas de Cacau) Ilhues/Itabuna Bahia Brazil and International clones were from CFC/ICCO/Biodiversity Clones, University of Reading, Reading, UK. (Table 1). 
Table 1. List of 58 cacao genotypes used for this experiment and obtained from 4 different origins maintained at ICT germplast bank.

\begin{tabular}{|c|c|c|c|c|c|c|c|c|c|c|c|c|}
\hline \multicolumn{3}{|c|}{$\begin{array}{l}\text { Wild cacao genotypes from River } \\
\text { basins of Peru Amazon (PWC) }\end{array}$} & \multicolumn{3}{|c|}{$\begin{array}{l}\text { Peruvian farmers' cacao genotypes } \\
\text { (PFC) }\end{array}$} & \multicolumn{4}{|c|}{ Brazilian genotypes (BCC) } & \multicolumn{2}{|c|}{$\begin{array}{l}\text { National } \quad \& \\
\text { genotypes (NIC) }\end{array}$} & \multirow{2}{*}{$\begin{array}{l}\text { International } \\
\text { Origin }\end{array}$} \\
\hline $\mathbf{N}^{\circ}$ & Genotype & Origin & $\mathbf{N}^{\circ}$ & Geno type & Origin & $\mathbf{N}^{\circ}$ & Genotype & Origin & & $\mathbf{N}^{\circ}$ & Genotype & \\
\hline 1 & AYP-15 & Aypena & 1 & ICT-1026 & $\begin{array}{l}\text { Mariscal Cáceres - } \\
\text { Juanjui }\end{array}$ & 1 & $\mathrm{BN}-34$ & Fazenda $\mathrm{E}$ & Nova & 1 & $\mathrm{CCN}-10$ & Ecuador \\
\hline 2 & AYP-20 & Aypena & 2 & ICT-1087 & $\begin{array}{l}\text { Mariscal Cáceres - } \\
\text { Juanjui }\end{array}$ & 2 & BS-01 & $\begin{array}{l}\text { Fazenda } \\
\text { Sossego }\end{array}$ & Bom & 2 & $\mathrm{CCN}-51$ & Ecuador \\
\hline 3 & AYP-22 & Aypena & 3 & ICT-1092 & $\begin{array}{l}\text { Mariscal Cáceres - } \\
\text { Juanjui }\end{array}$ & 3 & CA-14 & $\begin{array}{l}\text { Fazenda } \\
\text { Galo }\end{array}$ & Canta & 3 & EET-400 & Ecuador \\
\hline 4 & PAS-91 & Pastaza & 4 & ICT-1112 & $\begin{array}{l}\text { Mariscal Cáceres - } \\
\text { Juanjui }\end{array}$ & 4 & $\begin{array}{l}\text { CEPEC- } \\
2002\end{array}$ & $\begin{array}{l}\text { Centro de } \\
\text { do cacau }\end{array}$ & squisa & 4 & $\mathrm{H}-10$ & Peru \\
\hline 5 & PAS-93 & Pastaza & 5 & ICT-1189 & $\begin{array}{l}\text { Mariscal Cáceres - } \\
\text { Juanjui }\end{array}$ & 5 & CP-49-C10 & $\begin{array}{l}\text { Centro de } \\
\text { do cacau }\end{array}$ & squisa & 5 & ICS-1 & $\begin{array}{l}\text { Trinidad and } \\
\text { Tobago }\end{array}$ \\
\hline 6 & PAS-100 & Pastaza & 6 & ICT-1251 & $\begin{array}{l}\text { Mariscal Cáceres - } \\
\text { Juanjui }\end{array}$ & 6 & CP-53-C10 & $\begin{array}{l}\text { Centro de } \\
\text { do cacau }\end{array}$ & squisa & 6 & ICS-6 & $\begin{array}{l}\text { Trinidad and } \\
\text { Tobago }\end{array}$ \\
\hline 7 & PAS-105 & Pastaza & 7 & ICT-1281 & $\begin{array}{l}\text { Mariscal Cáceres - } \\
\text { Juanjui }\end{array}$ & 7 & $\begin{array}{l}\text { IPIRANGA- } \\
1\end{array}$ & Cidade de & ranga & 7 & ICS-39 & $\begin{array}{l}\text { Trinidad and } \\
\text { Tobago }\end{array}$ \\
\hline 8 & UGU-112 & Ungumayo & 8 & ICT-1292 & $\begin{array}{l}\text { Mariscal Cáceres - } \\
\text { Juanjui }\end{array}$ & 8 & $\mathrm{PH}-09$ & $\begin{array}{l}\text { Fazenda } \\
\text { Hibrido }\end{array}$ & Porto & 8 & ICS-95 & $\begin{array}{l}\text { Trinidad and } \\
\text { Tobago }\end{array}$ \\
\hline 9 & UGU-126 & Ungumayo & 9 & ICT-1506 & $\begin{array}{l}\text { Mariscal Cáceres - } \\
\text { Juanjui }\end{array}$ & 9 & $\mathrm{PH}-15$ & $\begin{array}{l}\text { Fazenda } \\
\text { Hibrido }\end{array}$ & Porto & 9 & IMC-67 & Peru \\
\hline 10 & UGU-130 & Ungumayo & 10 & ICT-2142 & Tocache & 10 & $\mathrm{PH}-16$ & $\begin{array}{l}\text { Fazenda } \\
\text { Hibrido }\end{array}$ & Porto & 10 & $\begin{array}{l}\text { POUND- } \\
12\end{array}$ & Peru \\
\hline 11 & UNG-53 & Ungurahui & 11 & ICT-2161 & Tocache & 11 & $\mathrm{PH}-17$ & $\begin{array}{l}\text { Fazenda } \\
\text { Hibrido }\end{array}$ & Porto & 11 & SCA-6 & Peru \\
\hline 12 & UNG-73 & Ungurahui & 12 & ICT-2171 & Tocache & 12 & $\mathrm{PH}-21$ & $\begin{array}{l}\text { Fazenda } \\
\text { Hibrido }\end{array}$ & Porto & 12 & TSH-565 & $\begin{array}{l}\text { Trinidad and } \\
\text { Tobago }\end{array}$ \\
\hline 13 & UNG-76 & Ungurahui & 13 & ICT-2172 & Tocache & 13 & $\mathrm{PH}-144$ & $\begin{array}{l}\text { Fazenda } \\
\text { Hibrido }\end{array}$ & Porto & 13 & TSH-1188 & $\begin{array}{l}\text { Trinidad and } \\
\text { Tobago }\end{array}$ \\
\hline \multirow[t]{2}{*}{14} & UNG-77 & Ungurahui & 14 & ICT-2173 & Tocache & 14 & PH-990 & $\begin{array}{l}\text { Fazenda } \\
\text { Hibrido }\end{array}$ & Porto & 14 & UF-613 & Costa Rica \\
\hline & & & 15 & ICT-2653 & Tocache & & & & & 15 & UF-667 & Costa Rica \\
\hline
\end{tabular}

\subsubsection{Shade (PPFD) levels}

The greenhouse was constructed and aligned in an east-west direction to evaluate the response of cacao genotypes to two levels of shade (Figure 1). The greenhouse was divided into two sections and covered with different light transmissibility plastic screens (Raschel mesh, from Arborizaciones EIRL ${ }^{\circledR}$ ) to achieve different levels of shade. The first section provided $80 \%$ shade with a PPFD of $400 \pm 50 \mu \mathrm{mol} \mathrm{m} \mathrm{m}^{-2} \mathrm{~s}^{-1}$, and the second section 
provided $50 \%$ shade with PPFD of $1000 \pm 50 \mu \mathrm{mol} \mathrm{m} \mathrm{m}^{-2} \mathrm{~s}^{-1}$. The PPFD in each section of the greenhouse was measured by a MQ-200 Quantum sensor (Apogee Instruments, Logan, UT USA). At both ends of greenhouse exhaust and inlet fans were installed to circulate air from inside to outside.

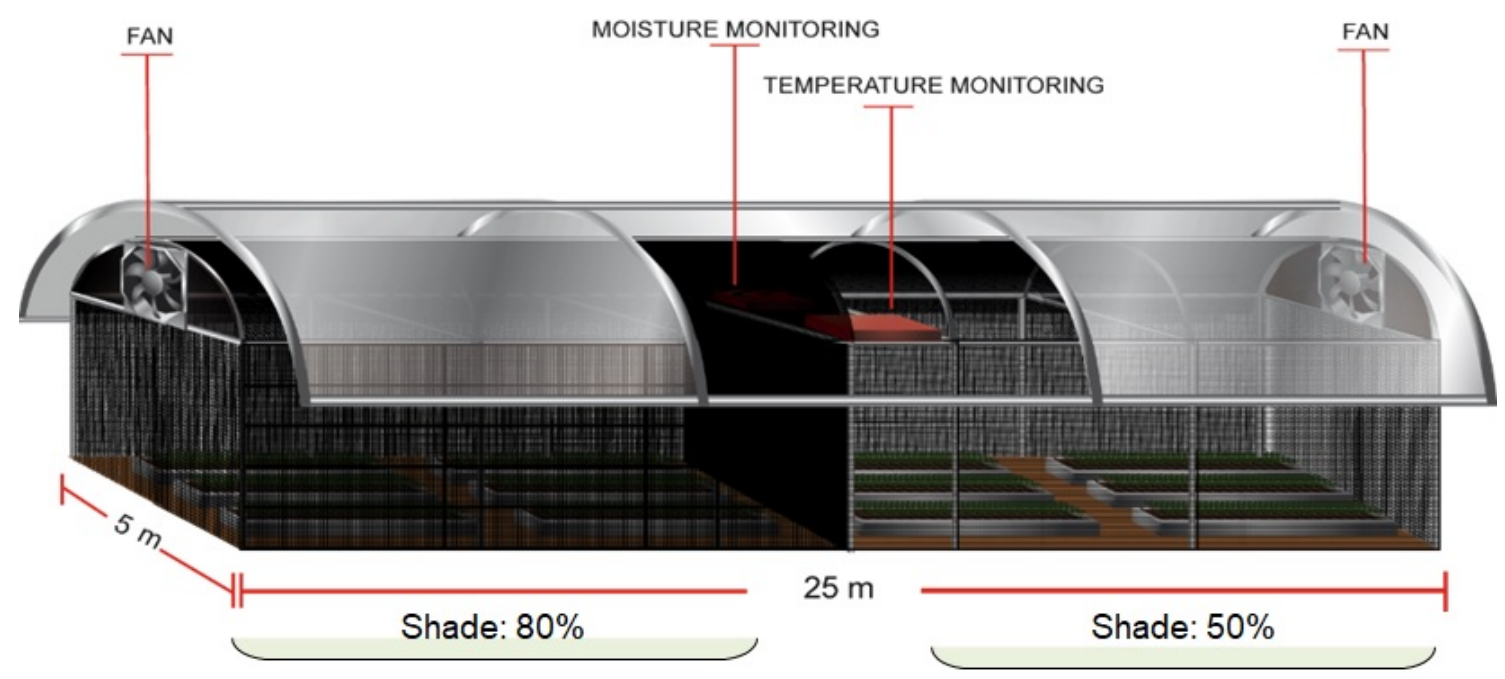

Figure 1. Greenhouse constructed at the ICT (Tropical Crops Institute) Tarapoto Peru to evaluate response of 58 cacao genotypes response to two levels of shade: 50\% (PPFD of $1000 \pm 50 \mu \mathrm{mol} \mathrm{m}^{-2} \mathrm{~s}^{-1}$ ) and $80 \%$ (PPFD of $400 \pm 50 \mu \mathrm{mol} \mathrm{m}^{-2} \mathrm{~s}^{-1}$ ).

\subsubsection{Plant growth conditions}

The rooted clonal cuttings from plagiotropic branches of various genotypes were prepared in the greenhouse. Terminal apical shoots with 3 or 4 leaves from each of the genotypes were cut, making a bevel cut, at the base of the branches $(3 / 4$ from the leaf area) and dipped into plant rooting hormone (Hormodin $3^{\circledR}, 0.8 \%$ indole-3-butyric acid, IBA) to induce roots. These cuttings were transplanted into polyethylene bags containing $2 \mathrm{~kg}$ of agricultural soil (sand $=50.96 \%$, silt $=22 \%$, clay $=27.04 \%$ ) previously fertilized with $60 \mathrm{~N}: 50 \mathrm{P}: 90 \mathrm{~K} \mathrm{~kg} \mathrm{ha}^{-1}$ applied as urea, calcium dihydrogen phosphate and potassium chloride respectively. Dolomitic lime $\left(1 \mathrm{MT} \mathrm{ha}^{-1}\right)$ was added to raise $\mathrm{pH}$ 6.0. Plants were maintained in the greenhouse at $28{ }^{\circ} \mathrm{C}, 80 \%$ relative humidity, with minimum light PPFD $50 \pm 5 \mu \mathrm{mol} \cdot \mathrm{m}^{-2} \cdot \mathrm{s}^{-1}$, soil moisture was maintained at the field capacity until root formation and proper pest control methods were adapted. At the end of 4 months growth, seedlings were transplanted into plastic pots containing $5 \mathrm{~kg}$ of sandy loam soil previously fertilized with urea (30 mg N kg$\left.{ }^{-1}\right), \mathrm{Ca}\left(\mathrm{H}_{2} \mathrm{PO}_{4}\right)_{2}\left(25 \mathrm{mg} \mathrm{P} \mathrm{kg}^{-1}\right), \mathrm{KCl}(45 \mathrm{mg} \mathrm{K}$ $\left.\mathrm{kg}^{-1}\right)$ and Dolomitic lime $\left(2.5 \mathrm{~g} \mathrm{~kg}^{-1}\right)$. Soil physicochemical properties of the sandy loam soil used for the study were: $72 \%$ sand, $18 \%$ clay, $1.55 \mathrm{~g} \mathrm{~cm}^{-3}$ bulk density, $58 \%$ porosity, $\mathrm{pH} 6.1$, organic matter $1.77 \%$, CEC $4.44 \mathrm{cmol} \mathrm{kg}^{-1}, \mathrm{~N}(0.08 \%), \mathrm{P}\left(4 \mathrm{mg} \mathrm{kg}^{-1}\right), \mathrm{K}(75 \mathrm{mg}$ 
$\left.\mathrm{kg}^{-1}\right), \mathrm{Ca}\left(1.22 \mathrm{cmolc}^{+} \mathrm{kg}^{-1}\right)$ and $\mathrm{Mg}\left(0.60 \mathrm{cmolc}^{+} \mathrm{kg}^{-1}\right)$, determined using the methods of Silva [51].

All the rooted genotypes seedlings were divided into two equal groups and subjected to two shade (PPFD) levels (Fig 1). Plants were grown for 6 months at different shades and soil moisture during growth was maintained at field capacity $(-33 \mathrm{KPa})$ by watering with deionized water every other day and soil moisture status was monitored with a soil moisture tensiometer (2724 ARL Jet Fill tensiometer, Soilmoisture Equipment Corp, Santa Barbara, CA USA). During growth of the plants, the greenhouse provided a mean temperature of $30.0{ }^{\circ} \mathrm{C}$ as well as relative humidity of $63 \%$. The experiment was conducted using a split-plot design with three replications under complete random distribution, two shade treatments were the main plots ( $50 \%$ and $80 \%$ shade) and 58 cacao genotypes were the subplots.

\subsection{Biometric parameters}

At the time of harvest, shoot length was measured from the base of the stem to the apex of the plant $(\mathrm{cm})$ and stem diameter was measured with a ruler and digital Vernier $(\mathrm{mm})$ respectively. At harvest stems, leaves and roots were separated; leaf and root area were measured in $\mathrm{cm}^{2}$ by image analysis (Assess 2.0: Image Analysis Software for Plant Disease Quantification, APS, Saint Paul, MN USA) [52]. All plant parts were washed with tap water, dipped in $1 \% \mathrm{HCl}$ and rinsed in distilled water, placed in paper sachets and oven dried at $60^{\circ} \mathrm{C}$ for 72 hours, until reaching a constant weight.

\subsection{Physiological parameters}

A week before harvest three mature leaves were selected randomly per genotype/pots and per shade treatment to record stomatal conductance (in $\mathrm{mmol} \mathrm{m}^{-2} \mathrm{~s}^{-1}$ ) using a SC-1 leaf porometer (Decagon Devices, Pullman, WA USA). Leaf chlorophyll content or "greenness" (in SPAD index) was measured by a chlorophyll meter, SPAD 502, (Spectrum Technologies, East Plainfield, IL USA).

Water use efficiency (WUE, g plant ${ }^{-1} \mathrm{~L}^{-1}$ ) was calculated as follows:

Eq.1. $W U E=\frac{S D W}{\text { Total water used during entire growth }}$ where:

SDW $=$ Shoot dry weight, g plant $^{-1}$

Total water used during entire growth period $=18.0 \mathrm{~L}(50 \%$ shade $)$ and $11.7 \mathrm{~L}(80 \%$ shade) plant ${ }^{-1}$ 


\subsection{Nutrient uptake parameters}

\subsubsection{Concentration of nutrients}

Oven dried shoots of all genotypes were ground to pass through a 1-mm sieve and 500 $\mathrm{mg}$ of sample was digested in $10 \mathrm{ml}$ of $65 \% \mathrm{HNO}_{3}$ [53] and the concentration of macronutrients $(\mathrm{K}, \mathrm{Ca}, \mathrm{Mg})$ and micronutrients $(\mathrm{Cu}, \mathrm{Fe}, \mathrm{Mn}$ and $\mathrm{Zn})$ in the digest were determined by atomic absorption spectrophotometry (AAS, Varian model "Spectra 55B", Victoria, Australia) and $\mathrm{P}$ by the ascorbic-molybdate color development method [51]. Total $\mathrm{N}$ was determined by the Kjeldahl method (digestion with $5 \mathrm{ml}$ of $\mathrm{H}_{2} \mathrm{SO}_{4} 95 \%$ ) both $\mathrm{N}$ and $\mathrm{P}$ were detected by a spectrophotometer (Spectronic 20D, Thermo Fisher, Waltham, MA USA) [51]. Concentrations are presented as the mean values from three replicates and expressed in $\mathrm{g} \mathrm{kg}^{-1}$ for macronutrients and $\mathrm{mg} \mathrm{kg}^{-1}$ for micronutrients.

\subsubsection{Nutrient uptake (U)}

The nutrient uptake $(U)$ or element content was calculated as follows:

Eq.2. $U=\frac{\text { Element concentration } \times S D W}{1000}$

where:

Element concentration $=$ in $\mathrm{g} \mathrm{kg}^{-1}$ (for macronutrients) or $\mathrm{mg} \mathrm{kg}^{-1}$ (for micronutrients)

SDW (Shoot dry weight $)=$ in g plant $^{-1}$

$\mathrm{U}$ (or content) $=\mathrm{g} \mathrm{plant}^{-1}$ (macronutrients) or $\mathrm{mg} \mathrm{plant}^{-1}$ (micronutrients)

\subsubsection{Nutrient uptake efficiency (NUE)}

Nutrient uptake efficiency (NUE), which is used to differentiate plant species, genotypes and cultivars for their ability to absorb and utilize nutrients for maximum yields [54, 55] was calculated as follows:

Eq.3. $N U E=\frac{1}{\text { Element concentration }} \times 1000$ where:

Element concentration $=$ in $\mathrm{g} \mathrm{kg}^{-1}$ for macronutrients and in $\mathrm{mg} \mathrm{kg}^{-1}$ for micronutrients NUE = in $\mathrm{g}$ shoot $\mathrm{g}^{-1}$ of any given macronutrient or in $\mathrm{g}$ shoot $\mathrm{mg}^{-1}$ of any given micronutrient

\subsection{Shade tolerance index (STI)}


Shade tolerance is the ability of a tree to survive and develop under light limited conditions [2]. To classify which cacao genotypes are tolerant or not tolerant, STI was calculated as described in the following equation:

Eq.4. $S T I=\frac{\text { Totol dry biomass at } 80 \% \text { shade }}{\text { Total dry biomass at } 50 \% \text { shade }} \times 100$

where:

Total (shoot + root) dry biomass $\left(\mathrm{g} \mathrm{plant}^{-1}\right.$ ) at $80 \%$ shade (PPFD $400 \mu \mathrm{mol} \mathrm{m}^{-2} \mathrm{~s}^{-1}$ ), this represents low light or heavy shade

Total (shoot + root) dry biomass $\left(\right.$ g plant $\left.^{-1}\right)$ at $50 \%$ shade (PPFD $1000 \mu \mathrm{mol} \mathrm{m}^{-2} \mathrm{~s}^{-1}$ ), this represents high light or low shade

Genotypes were classified into 3 groups: sensitive to shade (STI \% $\leq 40$ ), medium shade tolerant $(\mathrm{STI} \%>40$ but $\leq 60)$ and tolerant to shade $(\mathrm{STI} \%>60)$.

\subsection{Statistical analysis}

Statistical analyses of all the parameters, performed with Infostat ver. 2020 [56], consisted of a split-plot design under complete random distribution in order to compare means across two or more independent variables, in this case shade as a main plot and cacao genotypes as subplots. Normality and homogeneity of each parameter were tested by the Shapiro-Wilk test and Q-Q plot. When the effect of interactions between factors was not statistically significant $(\alpha>0.05)$ and the effect of a factor was significant, the analysis was extended to a Scott-Knott test used to compare means of each parameter.. Double bar graphs were made for NU and NUE, where the results of each nutrient was represented with comparisons between treatments: Shade-50\% and Shade- $80 \%$, average for each origin. For NUE the " $\mathrm{x}$ " axis was presented in a logarithmic scale to facilitate the vision of the results. "ns" indicated the results were not significant $(P>0.05)$ Standardized data were also assessed by Principal Component Analysis (PCA) using $R$ software version 1.2.5042, to evaluate the general correlations between biometric, physiological parameters and nutrient uptake (NU) of cacao seedlings for genotypes sensitive to shade and tolerant to $50 \%$ and $80 \%$ shade. 


\section{Results and Discussion}

Plants have the ability to adjust their morphology and physiology to a particular light environment. In the case of tropical and subtropical tree species, they have developed species-specific morphological and physiological features allowing them to optimize the capture of scarce solar radiation [36]. Larger leaf areas with anatomical properties associated with increased photosynthetic efficiency as well as an accumulation of anthocyanin are some of the responses of trees to low and high irradiance levels [36, 50], whereas root dry matter remains invariable, suggesting little anatomical or chemical changes [57].

Several authors have reported negative effects of shade on cacao growth $[30,58]$, whereas greater chlorophyll contents [7] and higher photosynthetic rates have been observed in plants under light shade but related to water regime, highlighting the genotypic differences as a response to shade factor [9,59]. The following sections summarize the results obtained in this study for 58 cacao genotypes subjected to $50 \%$ and $80 \%$ shade conditions.

\subsection{Growth parameters influenced by shade levels}

The interactions between cacao genotypes and shade levels for biometric parameters were significant $(P \leq 0.05)$, except for leaf area (Table 2$)$, but for this parameter, significant difference was found between the shade levels and across genotypes. These findings agree with a previous study conducted by Daymond et al. [60], where leaf area varied significantly in eight cacao clones (AMAZ 15/15, ICS-1, IMC-47, MAN 15/2, SC-1, SCA6, SPEC 54/1, UF-676) exposed to different levels of irradiance (0 to $696 \mu \mathrm{mol} \mathrm{m}^{-2} \mathrm{~s}^{-1}$ ). As highlighted by Acheampong et al. [9], genotypic differences in leaf area under the different shade levels suggest differential partitioning of assimilates in response to light in terms of final biomass.

In most cases, mean values of shoot length, leaf area, root area and shoot/root ratio per genotype were higher at $80 \%$ shade, while stem diameter, root dry weight and total dry biomass were higher at $50 \%$ shade. The minimum values of leaf and root area were observed for the AYP-20 genotype at 50 and $80 \%$ shade. For stem diameter the lowest value of $6.45 \mathrm{~mm}$ ( $80 \%$ shade) was recorded for the UGU-130 genotype. The minimum values of shoot length were 23.07 (50\% shade) and $33.07 \mathrm{~cm}(80 \%$ shade) for $\mathrm{PH}-09$ and CA-14 (BCC), respectively (Table 2). 
Table 2. Growth parameters (mean values per plant) of cacao genotypes from different origins subjected to two levels of shade $(50 \%$ and $80 \%)$.

\begin{tabular}{|c|c|c|c|c|c|c|c|c|c|c|c|c|c|c|}
\hline \multirow{2}{*}{ Genotype } & \multicolumn{2}{|c|}{$\mathrm{SL}(\mathrm{cm})$} & \multicolumn{2}{|c|}{$\mathrm{SD}(\mathrm{mm})$} & \multicolumn{2}{|c|}{$\mathrm{LA}\left(\mathrm{cm}^{2}\right)$} & \multicolumn{2}{|c|}{$\mathrm{RA}\left(\mathrm{cm}^{2}\right)$} & \multicolumn{2}{|c|}{ RDW (g) } & BDV & & $S / R$ & \\
\hline & $50 \%$ & $80 \%$ & $50 \%$ & $80 \%$ & $50 \%$ & $80 \%$ & $50 \%$ & $80 \%$ & $50 \%$ & $80 \%$ & $50 \%$ & $80 \%$ & $50 \%$ & $80 \%$ \\
\hline PWC & & & & & & & & & & & & & & \\
\hline$\overline{\text { AYP-15 }}$ & $33.33 c^{*}$ & $45.27 \mathrm{~b}$ & $10.72 b$ & $8.19 \mathrm{~d}$ & $706.92 b$ & $860.25 b$ & $239.83 d$ & $208.15 d$ & $3.75 \mathrm{e}$ & $1.14 \mathrm{e}$ & $25.49 d$ & $11.91 \mathrm{~g}$ & $6.22 \mathrm{~b}$ & $9.41 \mathrm{a}$ \\
\hline AYP-20 & $38.27 \mathrm{c}$ & $51.27 \mathrm{~b}$ & $10.84 \mathrm{~b}$ & $8.90 \mathrm{c}$ & $388.71 \mathrm{~b}$ & $701.45 b$ & $108.34 d$ & $151.63 d$ & $6.13 d$ & $2.64 \mathrm{e}$ & $30.97 \mathrm{c}$ & $16.03 \mathrm{f}$ & $4.07 \mathrm{c}$ & $5.25 \mathrm{~b}$ \\
\hline AYP-22 & $37.50 \mathrm{c}$ & $48.77 \mathrm{~b}$ & $8.94 \mathrm{c}$ & $7.88 \mathrm{~d}$ & $1245.41 a$ & $1032.92 a$ & $306.99 \mathrm{c}$ & $325.15 \mathrm{c}$ & $5.06 \mathrm{~d}$ & $1.94 \mathrm{e}$ & $26.02 \mathrm{~d}$ & $6.63 \mathrm{~g}$ & $4.39 \mathrm{~b}$ & $2.47 \mathrm{~d}$ \\
\hline PAS-100 & $27.67 \mathrm{c}$ & $36.80 \mathrm{c}$ & $9.91 \mathrm{c}$ & $7.49 \mathrm{~d}$ & $845.88 \mathrm{~b}$ & $959.96 \mathrm{~b}$ & $190.21 d$ & $222.01 \mathrm{~d}$ & $5.72 d$ & $1.93 \mathrm{e}$ & $29.19 d$ & $13.37 \mathrm{~g}$ & $4.48 \mathrm{~b}$ & $5.96 \mathrm{~b}$ \\
\hline PAS-105 & $32.67 \mathrm{c}$ & $36.13 c$ & $9.57 \mathrm{c}$ & $8.07 \mathrm{~d}$ & $596.09 b$ & $937.02 \mathrm{~b}$ & $300.61 \mathrm{c}$ & $388.76 \mathrm{~b}$ & $4.97 \mathrm{~d}$ & $1.40 \mathrm{e}$ & $28.44 d$ & $9.57 \mathrm{~g}$ & $4.74 \mathrm{~b}$ & $5.97 \mathrm{~b}$ \\
\hline PAS-91 & $31.63 c$ & $46.90 \mathrm{~b}$ & $11.84 a$ & $8.05 \mathrm{~d}$ & $1173.48 a$ & $1228.88 a$ & $290.09 c$ & $433.64 a$ & $10.71 \mathrm{~b}$ & $2.72 \mathrm{e}$ & $42.38 a$ & $13.33 \mathrm{~g}$ & $3.07 \mathrm{~d}$ & $4.19 c$ \\
\hline PAS-93 & $43.37 c$ & $59.97 a$ & $11.38 \mathrm{~b}$ & $8.39 \mathrm{~d}$ & $722.72 b$ & $1045.25 a$ & $197.86 d$ & $315.98 \mathrm{c}$ & $9.17 \mathrm{c}$ & $2.22 \mathrm{e}$ & $28.71 \mathrm{~d}$ & $12.68 \mathrm{~g}$ & $2.18 \mathrm{~d}$ & $4.71 \mathrm{~b}$ \\
\hline UGU-112 & $31.43 c$ & $44.37 \mathrm{~b}$ & $10.08 \mathrm{c}$ & $7.75 d$ & $531.56 \mathrm{~b}$ & $838.70 \mathrm{~b}$ & $239.69 d$ & $337.40 \mathrm{~b}$ & $6.92 \mathrm{c}$ & $1.31 \mathrm{e}$ & $29.12 d$ & $9.78 \mathrm{~g}$ & $3.93 c$ & $6.57 \mathrm{~b}$ \\
\hline UGU-126 & $30.57 \mathrm{c}$ & $41.03 \mathrm{c}$ & $10.97 \mathrm{~b}$ & $7.67 \mathrm{~d}$ & $733.08 \mathrm{~b}$ & $1057.4 a$ & $210.60 d$ & $281.22 \mathrm{c}$ & $5.91 \mathrm{~d}$ & $1.41 \mathrm{e}$ & $32.25 \mathrm{c}$ & $14.17 \mathrm{~g}$ & $4.71 \mathrm{~b}$ & $9.18 a$ \\
\hline UGU-130 & $23.37 \mathrm{c}$ & $35.77 \mathrm{c}$ & $8.64 d$ & $6.45 \mathrm{~d}$ & $666.84 \mathrm{~b}$ & $1152.7 a$ & $214.62 d$ & $209.03 d$ & $3.57 \mathrm{e}$ & $1.64 \mathrm{e}$ & $21.34 \mathrm{e}$ & $13.19 \mathrm{~g}$ & $5.19 b$ & $7.34 a$ \\
\hline UNG-53 & $35.60 \mathrm{c}$ & $42.87 \mathrm{c}$ & $9.09 \mathrm{c}$ & $7.64 d$ & $990.34 \mathrm{~b}$ & $1040.46 a$ & $251.17 \mathrm{c}$ & $361.67 \mathrm{~b}$ & $6.46 \mathrm{c}$ & $2.65 \mathrm{e}$ & $26.13 d$ & $11.37 \mathrm{~g}$ & $3.04 \mathrm{~d}$ & $3.27 \mathrm{c}$ \\
\hline UNG-73 & $31.83 c$ & $45.07 \mathrm{~b}$ & $10.63 b$ & $7.24 d$ & $658.56 \mathrm{~b}$ & $1235.49 a$ & $324.58 \mathrm{c}$ & $365.62 b$ & $9.76 \mathrm{~b}$ & $3.30 \mathrm{e}$ & $39.59 a$ & $18.07 f$ & $3.16 \mathrm{c}$ & $4.75 b$ \\
\hline UNG-76 & $36.60 \mathrm{c}$ & $49.33 b$ & $10.39 b$ & $7.10 \mathrm{~d}$ & $559.94 b$ & $944.88 \mathrm{~b}$ & $192.31 d$ & $192.97 d$ & $9.15 \mathrm{c}$ & $3.37 \mathrm{e}$ & $39.42 a$ & $21.75 \mathrm{e}$ & $3.42 \mathrm{c}$ & $5.48 \mathrm{~b}$ \\
\hline UNG-77 & $36.90 \mathrm{c}$ & $40.87 \mathrm{c}$ & $9.39 \mathrm{c}$ & $9.55 \mathrm{c}$ & $1108.74 a$ & $1300.43 a$ & $314.03 \mathrm{c}$ & $350.42 \mathrm{~b}$ & $9.93 \mathrm{~b}$ & $5.67 \mathrm{~d}$ & $32.28 \mathrm{c}$ & $21.70 \mathrm{e}$ & $2.32 \mathrm{~d}$ & $2.83 d$ \\
\hline PFC & & & & & & & & & & & & & & \\
\hline$\overline{\mathrm{ICT}}-1026$ & $45.00 \mathrm{~b}$ & $50.73 b$ & $12.43 a$ & $9.03 c$ & $749.85 b$ & $1246.95 a$ & $261.78 \mathrm{c}$ & $376.03 b$ & $10.41 b$ & $2.50 \mathrm{e}$ & $36.27 \mathrm{~b}$ & $11.78 \mathrm{~g}$ & $2.59 \mathrm{~d}$ & $4.49 \mathrm{~b}$ \\
\hline ICT-1087 & $45.87 \mathrm{~b}$ & $51.83 \mathrm{~b}$ & $12.63 a$ & $10.30 \mathrm{~b}$ & $904.52 \mathrm{~b}$ & $1102.92 a$ & $295.09 \mathrm{c}$ & $496.99 a$ & $10.04 \mathrm{~b}$ & $5.59 \mathrm{~d}$ & $41.94 a$ & $26.77 \mathrm{~d}$ & $3.22 \mathrm{c}$ & $3.87 \mathrm{c}$ \\
\hline ICT-1092 & $35.40 \mathrm{c}$ & $68.47 a$ & $13.00 a$ & $9.06 \mathrm{c}$ & $1040.10 a$ & $1202.26 a$ & $287.74 \mathrm{c}$ & $373.28 b$ & $9.03 c$ & $2.78 \mathrm{e}$ & $40.35 a$ & $17.19 f$ & $3.65 c$ & $5.29 b$ \\
\hline ICT-1112 & $42.27 \mathrm{c}$ & $55.10 a$ & $12.03 a$ & $7.88 \mathrm{~d}$ & $985.50 \mathrm{~b}$ & $1392.01 \mathrm{a}$ & $342.00 \mathrm{~b}$ & $391.44 b$ & $12.65 a$ & $5.37 \mathrm{~d}$ & $40.01 a$ & $20.91 \mathrm{e}$ & $2.16 \mathrm{~d}$ & $2.89 d$ \\
\hline ICT-1189 & $38.93 c$ & $50.67 \mathrm{~b}$ & $12.90 \mathrm{a}$ & $10.04 \mathrm{c}$ & $1207.02 a$ & $1253.8 \mathrm{a}$ & $292.81 \mathrm{c}$ & $341.51 \mathrm{~b}$ & $12.64 a$ & $4.31 \mathrm{~d}$ & $40.61 a$ & $20.04 \mathrm{e}$ & $2.22 \mathrm{~d}$ & $3.65 \mathrm{c}$ \\
\hline ICT-1251 & $38.53 \mathrm{c}$ & $51.60 \mathrm{~b}$ & $11.47 \mathrm{~b}$ & $9.72 \mathrm{c}$ & $812.41 \mathrm{~b}$ & $1162.32 a$ & $346.18 \mathrm{~b}$ & $416.42 a$ & $9.14 \mathrm{c}$ & $3.20 \mathrm{e}$ & $33.01 \mathrm{c}$ & $16.04 f$ & $2.62 \mathrm{~d}$ & $4.09 \mathrm{c}$ \\
\hline ICT-1281 & $36.93 \mathrm{c}$ & $37.27 \mathrm{c}$ & $11.02 \mathrm{~b}$ & $8.22 d$ & $656.73 b$ & $1221.94 a$ & $360.48 b$ & $379.81 \mathrm{~b}$ & $9.78 \mathrm{~b}$ & $2.59 \mathrm{e}$ & $34.20 \mathrm{~b}$ & $16.46 f$ & $2.54 \mathrm{~d}$ & $5.72 \mathrm{~b}$ \\
\hline ICT-1292 & $43.03 c$ & $54.90 a$ & $13.15 a$ & $9.02 \mathrm{c}$ & $968.91 b$ & $1057.95 a$ & $293.15 c$ & $371.26 \mathrm{~b}$ & $12.05 a$ & $3.96 \mathrm{e}$ & $37.69 \mathrm{~b}$ & $15.65 \mathrm{~g}$ & $2.16 \mathrm{~d}$ & $3.03 d$ \\
\hline ICT-1506 & $38.97 \mathrm{c}$ & $49.00 \mathrm{~b}$ & $11.42 \mathrm{~b}$ & $10.88 \mathrm{~b}$ & $776.12 \mathrm{~b}$ & $1038.42 a$ & $304.42 \mathrm{c}$ & $460.27 a$ & $9.42 \mathrm{~b}$ & $3.67 \mathrm{e}$ & $31.95 \mathrm{c}$ & $21.17 \mathrm{e}$ & $2.48 \mathrm{~d}$ & $4.77 \mathrm{~b}$ \\
\hline ICT-2142 & $34.90 \mathrm{c}$ & $46.20 \mathrm{~b}$ & $11.20 \mathrm{~b}$ & $9.26 \mathrm{c}$ & $776.68 \mathrm{~b}$ & $822.84 \mathrm{~b}$ & $322.66 \mathrm{c}$ & $449.18 a$ & $10.17 \mathrm{~b}$ & $3.37 \mathrm{e}$ & $28.95 \mathrm{~d}$ & $19.44 \mathrm{e}$ & $1.87 \mathrm{~d}$ & $4.89 \mathrm{~b}$ \\
\hline ICT-2161 & $35.00 \mathrm{c}$ & $46.10 \mathrm{~b}$ & $11.78 a$ & $10.13 c$ & $616.93 b$ & $983.81 \mathrm{~b}$ & $257.92 \mathrm{c}$ & $472.67 a$ & $10.04 \mathrm{~b}$ & $3.60 \mathrm{e}$ & $32.34 \mathrm{c}$ & $16.35 \mathrm{f}$ & $2.22 \mathrm{~d}$ & $3.88 \mathrm{c}$ \\
\hline ICT-2171 & $41.53 c$ & $55.10 a$ & $13.27 a$ & $8.58 \mathrm{~d}$ & $881.30 \mathrm{~b}$ & $999.13 a$ & $285.55 \mathrm{c}$ & $336.09 \mathrm{~b}$ & $12.46 a$ & $5.54 d$ & $35.91 \mathrm{~b}$ & $22.19 \mathrm{e}$ & $1.89 \mathrm{~d}$ & $3.00 \mathrm{~d}$ \\
\hline ICT-2172 & $37.60 \mathrm{c}$ & $65.43 a$ & $13.65 a$ & $8.70 \mathrm{~d}$ & $1437.02 a$ & $1412.61 a$ & $249.74 \mathrm{c}$ & $307.74 \mathrm{c}$ & $12.27 \mathrm{a}$ & $4.64 d$ & $34.35 \mathrm{~b}$ & $22.83 \mathrm{e}$ & $1.80 \mathrm{~d}$ & $3.92 \mathrm{c}$ \\
\hline ICT-2173 & $47.40 \mathrm{~b}$ & $48.93 b$ & $10.27 b$ & $8.49 d$ & $785.07 \mathrm{~b}$ & $1255.67 a$ & $277.44 \mathrm{c}$ & $349.77 \mathrm{~b}$ & $7.54 \mathrm{c}$ & $4.18 d$ & $28.69 d$ & $21.09 \mathrm{e}$ & $2.98 \mathrm{~d}$ & $4.04 \mathrm{c}$ \\
\hline $\begin{array}{l}\text { ICT-2653 } \\
\text { BCC }\end{array}$ & $32.10 \mathrm{c}$ & $47.23 b$ & $11.93 a$ & $9.76 c$ & $879.42 b$ & $1035.99 a$ & $337.94 b$ & $307.94 c$ & $11.57 a$ & $3.67 \mathrm{e}$ & $37.41 \mathrm{~b}$ & $13.43 \mathrm{~g}$ & $2.24 d$ & $2.67 d$ \\
\hline $\mathrm{BN}-34$ & $37.70 \mathrm{c}$ & $35.57 \mathrm{c}$ & $8.54 d$ & $6.65 \mathrm{~d}$ & $763.10 \mathrm{~b}$ & $1058.05 a$ & $334.53 b$ & $340.96 b$ & $9.25 c$ & $3.63 \mathrm{e}$ & $32.60 \mathrm{c}$ & $16.18 \mathrm{f}$ & $2.53 d$ & $3.49 c$ \\
\hline BS-01 & $34.50 \mathrm{c}$ & $41.73 c$ & $8.72 \mathrm{~d}$ & $9.83 c$ & $879.54 \mathrm{~b}$ & 1079.15a & $338.36 \mathrm{~b}$ & $402.63 a$ & $7.60 \mathrm{c}$ & $3.38 \mathrm{e}$ & $31.68 \mathrm{c}$ & $15.64 \mathrm{~g}$ & $3.29 \mathrm{c}$ & $3.63 \mathrm{c}$ \\
\hline CA-14 & $36.73 c$ & $33.07 \mathrm{c}$ & $9.65 c$ & $7.66 \mathrm{~d}$ & $818.76 \mathrm{~b}$ & $834.93 b$ & $272.47 \mathrm{c}$ & $361.36 \mathrm{~b}$ & $6.47 \mathrm{c}$ & $2.49 \mathrm{e}$ & $26.66 \mathrm{~d}$ & $17.44 \mathrm{f}$ & $3.43 c$ & $6.07 \mathrm{~b}$ \\
\hline CEPEC-2002 & $36.20 \mathrm{c}$ & $40.43 c$ & $10.72 \mathrm{~b}$ & $8.25 \mathrm{~d}$ & $632.53 b$ & $970.48 \mathrm{~b}$ & $302.44 \mathrm{c}$ & $288.82 \mathrm{c}$ & $7.95 \mathrm{c}$ & $3.27 \mathrm{e}$ & $29.00 \mathrm{~d}$ & $10.96 \mathrm{~g}$ & $2.60 d$ & $2.31 \mathrm{~d}$ \\
\hline CP-49-C10 & $36.27 \mathrm{c}$ & $35.23 c$ & $10.52 \mathrm{~b}$ & $7.58 d$ & $763.34 \mathrm{~b}$ & $1118.17 a$ & $332.71 \mathrm{~b}$ & $390.72 b$ & $7.69 \mathrm{c}$ & $3.00 \mathrm{e}$ & $34.63 \mathrm{~b}$ & $12.96 \mathrm{~g}$ & $3.51 \mathrm{c}$ & $3.37 \mathrm{c}$ \\
\hline CP-53-C10 & $31.40 \mathrm{c}$ & $37.13 c$ & $9.79 \mathrm{c}$ & $7.62 \mathrm{~d}$ & $812.02 \mathrm{~b}$ & $950.53 \mathrm{~b}$ & $290.11 \mathrm{c}$ & $343.77 \mathrm{~b}$ & $7.25 \mathrm{c}$ & $2.62 \mathrm{e}$ & $32.38 \mathrm{c}$ & $11.66 \mathrm{~g}$ & $3.64 \mathrm{c}$ & $3.60 \mathrm{c}$ \\
\hline IPIRANGA-1 & $31.73 c$ & $56.00 a$ & $10.05 \mathrm{c}$ & $7.93 d$ & 1117.13a & $1166.97 a$ & $340.01 \mathrm{~b}$ & $345.07 \mathrm{~b}$ & $7.41 \mathrm{c}$ & $2.87 \mathrm{e}$ & $30.37 \mathrm{c}$ & $10.88 \mathrm{~g}$ & $3.62 \mathrm{c}$ & $3.71 \mathrm{c}$ \\
\hline PH-09 & $23.07 \mathrm{c}$ & $46.63 b$ & $9.92 \mathrm{c}$ & $8.33 d$ & $687.77 \mathrm{~b}$ & $1002.07 a$ & $307.15 c$ & $270.79 c$ & $8.20 c$ & $1.30 \mathrm{e}$ & $33.86 \mathrm{~b}$ & $9.39 \mathrm{~g}$ & $3.19 c$ & $6.54 \mathrm{~b}$ \\
\hline PH-144 & $45.33 b$ & $54.07 a$ & $11.99 a$ & $9.63 \mathrm{c}$ & $977.80 \mathrm{~b}$ & $1016.84 a$ & $252.06 \mathrm{c}$ & $343.32 b$ & $7.66 \mathrm{c}$ & $3.03 \mathrm{e}$ & $34.97 \mathrm{~b}$ & $21.61 \mathrm{e}$ & $3.59 \mathrm{c}$ & $6.19 b$ \\
\hline $\mathrm{PH}-15$ & $34.73 \mathrm{c}$ & $51.40 \mathrm{~b}$ & $9.79 c$ & $8.56 \mathrm{~d}$ & $872.39 \mathrm{~b}$ & $969.05 \mathrm{~b}$ & $439.71 a$ & $450.62 a$ & $5.22 \mathrm{~d}$ & $2.55 \mathrm{e}$ & $25.58 \mathrm{~d}$ & $13.40 \mathrm{~g}$ & $4.01 \mathrm{c}$ & $4.33 \mathrm{~b}$ \\
\hline PH-16 & $29.53 \mathrm{c}$ & $34.07 \mathrm{c}$ & $9.62 \mathrm{c}$ & $7.53 d$ & $817.56 \mathrm{~b}$ & $980.65 \mathrm{~b}$ & $368.82 \mathrm{~b}$ & $371.42 \mathrm{~b}$ & $9.13 \mathrm{c}$ & $3.21 \mathrm{e}$ & $29.15 d$ & $14.06 \mathrm{~g}$ & $2.29 \mathrm{~d}$ & $3.36 \mathrm{c}$ \\
\hline $\mathrm{PH}-17$ & $31.20 \mathrm{c}$ & $35.57 \mathrm{c}$ & $9.09 \mathrm{c}$ & $7.16 \mathrm{~d}$ & $801.06 \mathrm{~b}$ & $1115.94 a$ & $251.80 \mathrm{c}$ & $257.28 \mathrm{c}$ & $7.50 \mathrm{c}$ & $3.52 \mathrm{e}$ & $25.56 \mathrm{~d}$ & $12.82 \mathrm{~g}$ & $2.48 \mathrm{~d}$ & $2.65 d$ \\
\hline PH-21 & $39.97 \mathrm{c}$ & $53.20 a$ & $9.34 \mathrm{c}$ & $8.27 \mathrm{~d}$ & $791.30 \mathrm{~b}$ & 1077.63a & $302.73 \mathrm{c}$ & $221.90 \mathrm{~d}$ & $4.24 d$ & $3.06 \mathrm{e}$ & $26.84 d$ & $17.85 f$ & $5.33 b$ & $4.87 \mathrm{~b}$ \\
\hline PH-990 & $35.47 \mathrm{c}$ & $40.30 \mathrm{c}$ & $9.41 \mathrm{c}$ & $7.83 \mathrm{~d}$ & $943.25 \mathrm{~b}$ & $1185.36 a$ & $318.69 \mathrm{c}$ & $332.32 b$ & $5.43 d$ & $2.99 \mathrm{e}$ & $27.45 d$ & $17.21 \mathrm{f}$ & $4.73 b$ & $5.09 b$ \\
\hline NIC & & & & & & & & & & & & & & \\
\hline$\overline{\mathrm{CCN}}-10$ & $31.27 \mathrm{c}$ & $41.33 \mathrm{c}$ & $13.43 a$ & $10.48 b$ & $1024.44 a$ & $1184.12 a$ & $298.51 \mathrm{c}$ & $442.30 a$ & $11.02 \mathrm{a}$ & $3.63 e$ & $34.39 \mathrm{~b}$ & $17.13 \mathrm{f}$ & $2.14 d$ & $3.77 \mathrm{c}$ \\
\hline CCN-51 & $40.10 \mathrm{c}$ & $45.33 \mathrm{~b}$ & $12.06 a$ & $10.56 \mathrm{~b}$ & $1294.43 a$ & $1393.24 a$ & $404.42 a$ & $494.13 a$ & $11.30 a$ & $5.70 \mathrm{~d}$ & $40.18 a$ & $22.28 \mathrm{e}$ & $2.61 \mathrm{~d}$ & $2.91 \mathrm{~d}$ \\
\hline EET-400 & $39.27 \mathrm{c}$ & $50.03 \mathrm{~b}$ & $10.93 \mathrm{~b}$ & $8.55 \mathrm{~d}$ & $965.54 \mathrm{~b}$ & $988.93 \mathrm{~b}$ & $240.07 d$ & $308.90 \mathrm{c}$ & $9.76 \mathrm{~b}$ & $3.07 \mathrm{e}$ & $32.60 \mathrm{c}$ & $17.21 \mathrm{f}$ & $2.38 \mathrm{~d}$ & $4.69 \mathrm{~b}$ \\
\hline $\mathrm{H}-10$ & $50.05 \mathrm{~b}$ & $63.10 a$ & $12.26 a$ & $8.05 \mathrm{~d}$ & $720.73 b$ & $1335.75 a$ & $474.94 a$ & $372.16 \mathrm{~b}$ & $8.15 \mathrm{c}$ & $3.81 \mathrm{e}$ & $27.16 \mathrm{~d}$ & $13.09 \mathrm{~g}$ & $2.31 \mathrm{~d}$ & $2.52 \mathrm{~d}$ \\
\hline ICS-1 & $41.30 \mathrm{c}$ & $42.93 c$ & $12.14 a$ & $9.32 \mathrm{c}$ & $917.33 b$ & $1140.95 a$ & $272.13 \mathrm{c}$ & $407.49 a$ & $8.67 \mathrm{c}$ & $4.18 d$ & $29.37 d$ & $22.22 \mathrm{e}$ & $2.38 \mathrm{~d}$ & $4.35 \mathrm{~b}$ \\
\hline ICS-39 & $41.47 \mathrm{c}$ & $53.90 a$ & $12.40 \mathrm{a}$ & $8.53 \mathrm{~d}$ & $816.56 \mathrm{~b}$ & $1054.92 a$ & $305.41 \mathrm{c}$ & $372.95 b$ & $7.33 \mathrm{c}$ & $3.34 \mathrm{e}$ & $32.80 \mathrm{c}$ & $20.93 \mathrm{e}$ & $3.53 \mathrm{c}$ & $5.63 \mathrm{~b}$ \\
\hline ICS-6 & $36.40 \mathrm{c}$ & $45.57 \mathrm{~b}$ & $12.14 a$ & $8.21 \mathrm{~d}$ & $801.38 \mathrm{~b}$ & $914.53 \mathrm{~b}$ & $328.02 \mathrm{c}$ & $453.16 a$ & $7.56 \mathrm{c}$ & $3.91 \mathrm{e}$ & $29.79 \mathrm{~d}$ & $15.83 \mathrm{~g}$ & $2.96 \mathrm{~d}$ & $3.06 \mathrm{~d}$ \\
\hline ICS-95 & $44.80 \mathrm{~b}$ & $37.93 c$ & $11.03 \mathrm{~b}$ & $8.32 \mathrm{~d}$ & $967.35 \mathrm{~b}$ & $1182.73 a$ & $284.28 \mathrm{c}$ & $345.16 \mathrm{~b}$ & $8.20 \mathrm{c}$ & $2.63 \mathrm{e}$ & $31.51 \mathrm{c}$ & $15.46 \mathrm{~g}$ & $2.86 \mathrm{~d}$ & $5.09 \mathrm{~b}$ \\
\hline IMC-67 & $42.27 \mathrm{c}$ & $44.83 \mathrm{~b}$ & $13.49 a$ & $8.78 \mathrm{c}$ & $1022.91 \mathrm{a}$ & $1281.34 a$ & $364.60 \mathrm{~b}$ & $472.07 a$ & $11.37 a$ & $3.07 \mathrm{e}$ & $33.77 \mathrm{~b}$ & $14.30 \mathrm{~g}$ & $2.07 \mathrm{~d}$ & $3.75 \mathrm{c}$ \\
\hline POUND-12 & $42.07 \mathrm{c}$ & $45.97 \mathrm{~b}$ & $12.00 \mathrm{a}$ & $9.55 \mathrm{c}$ & $1038.90 a$ & $1117.07 a$ & $341.77 \mathrm{~b}$ & $452.85 a$ & $7.03 c$ & $3.80 \mathrm{e}$ & $28.47 \mathrm{~d}$ & $17.28 \mathrm{f}$ & $3.07 \mathrm{~d}$ & $3.56 c$ \\
\hline SCA-6 & $35.77 \mathrm{c}$ & $39.83 c$ & $11.01 \mathrm{~b}$ & $10.22 \mathrm{~b}$ & $943.69 b$ & $1315.00 \mathrm{a}$ & $269.91 \mathrm{c}$ & $412.50 a$ & $7.27 \mathrm{c}$ & $2.80 \mathrm{e}$ & $28.18 d$ & $13.68 \mathrm{~g}$ & $2.98 \mathrm{~d}$ & $4.15 \mathrm{c}$ \\
\hline TSH-1188 & $35.83 \mathrm{c}$ & $44.40 \mathrm{~b}$ & $12.36 a$ & $8.74 \mathrm{c}$ & $737.38 \mathrm{~b}$ & $1054.80 a$ & $345.92 \mathrm{~b}$ & $430.10 a$ & $10.29 b$ & $3.49 \mathrm{e}$ & $32.50 \mathrm{c}$ & $17.62 \mathrm{f}$ & $2.19 d$ & $4.10 \mathrm{c}$ \\
\hline TSH-565 & $33.77 \mathrm{c}$ & $42.07 \mathrm{c}$ & $11.86 a$ & $9.17 \mathrm{c}$ & $902.88 \mathrm{~b}$ & $1262.33 a$ & $289.53 \mathrm{c}$ & $433.21 a$ & $9.52 \mathrm{~b}$ & $3.00 \mathrm{e}$ & $35.75 \mathrm{~b}$ & $12.18 \mathrm{~g}$ & $2.75 \mathrm{~d}$ & $4.10 \mathrm{c}$ \\
\hline UF-613 & $33.27 \mathrm{c}$ & $38.87 \mathrm{c}$ & $11.79 a$ & $8.36 \mathrm{~d}$ & $815.09 b$ & $1070.66 \mathrm{a}$ & $303.36 \mathrm{c}$ & $442.48 a$ & $7.51 \mathrm{c}$ & $3.77 \mathrm{e}$ & $28.87 \mathrm{~d}$ & $18.53 \mathrm{f}$ & $2.85 \mathrm{~d}$ & $3.95 \mathrm{c}$ \\
\hline UF-667 & $38.17 \mathrm{c}$ & $43.33 c$ & $11.63 \mathrm{~b}$ & $8.63 d$ & $1319.35 a$ & $1527.33 a$ & $395.79 \mathrm{~b}$ & $434.70 \mathrm{a}$ & $10.39 \mathrm{~b}$ & $3.80 \mathrm{e}$ & $35.91 \mathrm{~b}$ & $19.70 \mathrm{e}$ & $2.52 d$ & $4.21 \mathrm{c}$ \\
\hline Media PWC & $33.62 d$ & $44.60 \mathrm{~b}$ & $10.17 \mathrm{~b}$ & $7.88 \mathrm{~d}$ & $780.59 d$ & $1023.99 b$ & $241.50 \mathrm{~d}$ & $295.97 c$ & $6.94 \mathrm{c}$ & $2.38 \mathrm{e}$ & $30.81 \mathrm{~b}$ & $13.83 d$ & $3.92 \mathrm{~b}$ & $5.53 a$ \\
\hline Media PFC & $39.56 \mathrm{c}$ & $51.90 a$ & $12.14 a$ & $9.27 \mathrm{c}$ & $898.51 \mathrm{c}$ & $1145.91 a$ & $300.99 \mathrm{c}$ & $388.69 a$ & $10.61 a$ & $3.93 d$ & $35.58 a$ & $18.76 \mathrm{c}$ & $2.44 d$ & $4.01 \mathrm{~b}$ \\
\hline Media BCC & $34.56 \mathrm{~d}$ & $42.46 \mathrm{~b}$ & $9.80 \mathrm{~b}$ & $8.06 \mathrm{~d}$ & $834.11 \mathrm{~d}$ & $1037.56 \mathrm{~b}$ & $317.97 \mathrm{~b}$ & $337.21 \mathrm{~b}$ & $7.21 \mathrm{c}$ & $2.92 \mathrm{e}$ & $30.05 \mathrm{~b}$ & $14.43 d$ & $3.45 \mathrm{c}$ & $4.23 \mathrm{~b}$ \\
\hline Media NIC & $39.05 \mathrm{c}$ & $45.30 \mathrm{~b}$ & $12.04 a$ & $9.03 c$ & $952.53 c$ & $1188.25 a$ & $327.91 b$ & $418.28 a$ & $9.02 b$ & $3.60 \mathrm{~d}$ & $32.08 \mathrm{~b}$ & $17.16 \mathrm{c}$ & $2.64 \mathrm{~d}$ & $3.99 \mathrm{~b}$ \\
\hline Media Total & $36.79 \mathrm{~B}^{\star *}$ & $46.15 A$ & $11.07 \mathrm{~A}$ & $8.58 \mathrm{~B}$ & 868.47B & $1110.27 \mathrm{~A}$ & 297.69B & $361.54 \mathrm{~A}$ & $8.50 \mathrm{~A}$ & $3.23 \mathrm{~B}$ & $32.19 \mathrm{~A}$ & 16.11B & $3.09 \mathrm{~B}$ & $4.42 \mathrm{~A}$ \\
\hline $\begin{array}{l}\text { Source of } \\
\text { variation }\end{array}$ & & & $F$ & & & & & & & & & & & \\
\hline Shade (S) & 0.0004 & & 0.0001 & & 0.0001 & & $<0.0001$ & & $<0.0001$ & & $<0.0001$ & & 0.0001 & \\
\hline Genotype (G) 57 & $<0.0001$ & & $<0.0001$ & & $<0.0001$ & & $<0.0001$ & & $<0.0001$ & & $<0.0001$ & & $<0.0001$ & \\
\hline$G^{*} S$ & 0.0072 & & 0.0003 & & 0.6657 & & 0.0008 & & $<0.0001$ & & $<0.0001$ & & 0.0006 & \\
\hline
\end{tabular}

SL: shoot length, SD: stem diameter, LA: leaf area, RA: root area, RDW: root dry weight, BDW: total dry weight, S/R: shoot/root rate. $\left.{ }^{*}\right)$ Different lower case letters on the right of each value in both columns of each variable indicate a significant difference between genotypes and shade level, (Scott $\&$ Knott test, $P \leq 0.05)$. ( $\left.{ }^{* *}\right)$ Different capital letters on the right of each value in line of each variable indicate a significant difference between shade level (Scott \& Knott test, $P \leq 0.05)$. PWC: wild cacao from river basins of Peruvian Amazon collection, PFC: Peruvian farmers' collection, BCC: Brazilian cacao collection, NIC: National and International cacao collection. Different lower case letters in parenthesis on the right of each value in both columns of each variable indicate a significant difference between genotypes and shade level, (Scott \& Knott test, $(P \leq 0.05)$ 
As reported by Galyuon et al. [12], cacao grown at full sunlight $\left(1800 \mu \mathrm{mol} \mathrm{m}^{-2} \mathrm{~s}^{-1}\right)$ and shade $\left(900 \mu \mathrm{mol} \mathrm{m} \mathrm{m}^{-2} \mathrm{~s}^{-1}\right)$ had different morphology. At full sunlight, leaf size, internode length, total leaf area and dry matter per plant were significantly reduced, while leaf thickness and leaf number per plant were increased compared to plants grown in shade. Besides, cacao plants growing between 35 and 55\% shade have a leaf area higher than those grown under heavy shade whereas stem dry matter accumulation was lower as the level of shade increased [9].

Da Silva Branco et al. [10] evaluated the effects of four levels of shade (no shade, $50 \%$ $29 \%$ and $22 \%$ of the incident of radiation) and two levels of water regime (control and flooded plants) on cacao seedlings performance. They reported that responses of shoot length, stem diameter, leaf number, total leaf area, specific leaf area, root biomass and total biomass varied by genotype, treatment or combined effects. For example, leaf number and leaf area were reduced by increasing light intensity, on the other hand root length and collar diameter didn't vary when exposed to different shade levels.

Similarly, stem diameter can be wider in cacao monocultures, followed by agroforestry and successional agroforestry systems in a long-term field trial in Alto Beni, Bolivia [18]. Nevertheless, the production system (full-sun monoculture, agroforestry system, both under organic and conventional farming, and a highly diverse successional agroforestry system under organic farming) didn't have an effect on root length, surface area, specific root length, specific root area or diameter [61]. But in the highly successional agroforestry system, root volume and biomass were higher than those found in the agroforestry system [61]. These results are in agreement with our findings (Table 2). An investment in root growth is most relevant in plants under high rather than low light intensity to provide large surface for evapotranspiration and maintain cell turgor pressure [10].

A maximum shoot/root ratio was recorded for the AYP-15 cacao genotype (PWC) for both shade levels. The highest values of root dry weight 12.65 and $5.70 \mathrm{~g} \mathrm{plant}^{-1}$ were noted for the genotypes ICT-1112 (PFC) and CCN-51 (NIC) at 50\% and $80 \%$ shade, respectively. The PAS-91 genotype had the highest total dry weight-- with $42.38 \mathrm{~g} \mathrm{plant}^{-}$ ${ }^{1}$ (50\% shade) and ICT-1087 with 26.77 g plant $^{-1}$ (80\% shade) (Table 2). Baligar et al. [43] observed an increase in biomass accumulation in cacao roots, leaves, stems and shoots in a greenhouse experiment when PPFD increased from 65 to $190 \mu \mathrm{mol} \mathrm{m} \mathrm{s}^{-1}$ (high to medium shade) that also entailed a reduction in leaf area and leaf specific area. Recently Baligar et al. [44] reported that in seven genetically different cacao genotypes increasing PPFD from 100 to $400 \mu \mathrm{mol} \mathrm{m}^{-2} \mathrm{~s}^{-1}$ increased shoot and root growth, relative growth rate (RGR) and net assimilation rates (NAR). Moreover, cacao grown under two shaded systems, one with $\mathrm{N}$-fixing legume trees and one with different shade trees, 
compared to a monoculture system, had higher stem density, shoot:root ratio, aboveground and root biomass but similar tree height and a lower stem diameter [19].

\subsection{Physiological parameters influenced by shade}

The interaction between cacao genotypes and shade levels was significant for the physiological parameters (Tables 3).

An increase in chlorophyll content (SPAD index) was observed in cacao seedlings under $80 \%$ shade compared to $50 \%$ shade, with some exceptions across genotypes. By contrast, overall stomatal conductance was higher in plants grown at $50 \%$ shade, however there were some exceptions in genotypes of the PFC and NIC (Table 3)

The maximum level of chlorophyll content at $50 \%$ shade was 34.70 SPAD for UGU-130 genotype, whereas at $80 \%$ shade it was 34.50 SPAD for AYP-15, both from the PWC. The range of observed stomatal conductance was between 104.57 and $363.03 \mathrm{mmol} \mathrm{m}^{-}$ ${ }^{2} \mathrm{~s}^{-1}$ at $50 \%$ shade for UGU-112 and $\mathrm{PH}-21$ respectively, and between 47.33 and 260.67 mmol m $\mathrm{m}^{-2} \mathrm{~s}^{-1}$ at $80 \%$ shade for UGU-126 and $\mathrm{H}-10$, respectively (Table 3 ).

With respect to chlorophyll content, shade leaves in cacao often exhibit greater total chlorophyll concentrations per unit mass than sun leaves. De Almeida and Valle [62] and Daymond and Hadley [59] reported a consistent decline in chlorophyll content with increasing light in three cacao genotypes and it can vary quite considerably over time. Moreover, Acheampong et al. [63], reported that no clear pattern was observed for leaf chlorophyll in cacao genotypes grown under three shade levels provided by plants and under fertilizer application. Our results show either a reduction of leaf chlorophyll content while PPFD increases or little variation between shade levels (Table 3 ).

Acheampong et al. [9] conducted a study with four cacao genotypes (T 79/501, PA-150, SCA-6 and P-30) and subjected them to three shade levels (32.5, 55 and $76 \%$ ) at two seasons of growth (dry and rainy). They reported that stomatal conductance was higher in seedlings under heavy shade during the dry season and lower in the rainy season for seedlings under lighter shade. As a consequence, photosynthesis rates were higher in the wet season for cacao under medium and light shade. Results from one crop year for the same genotypes grown under three shade levels provided by plants and under fertilizer application, showed that a higher stomatal conductance was related to an increase in shade but only for two genotypes [63]. However, the opposite trend is observed in our study where stomatal conductance decreases with higher shade considering the overall results, as well as specific values for SCA-6 cacao genotype (Table 3). Overall stomatal conductance was higher in plants grown at low shade $(50 \%$ shade), however there were some exceptions in genotypes of the PFC and the NIC (Table 3). 
Table 3. Physiological parameters (mean values)- and Shade Tolerance Index (STI) for 58 cacao genotypes subjected to two levels of shade (50 and $80 \%$ )

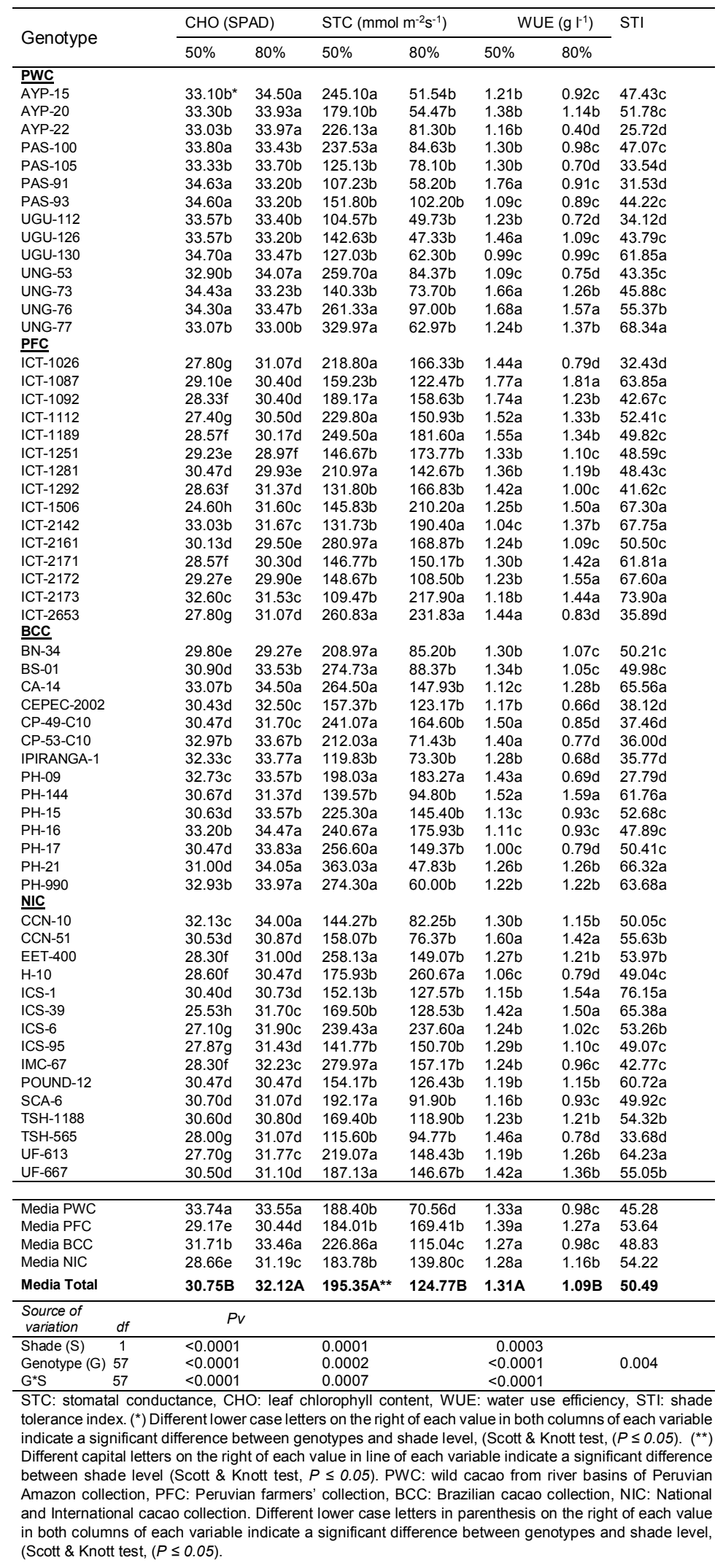


The evaluation of stomatal conductance in nine cacao genotypes (TCS-13, TCS-19, SCC-53, SCC-82, SCC-83, CCS-73, CCS-77, CCS-80 and ICS-95) grown in two agroforestry systems in Colombia with a maximum irradiance of 2100 and $1800 \mu \mathrm{mol} \mathrm{m}$ ${ }^{2} \mathrm{~s}^{-1}$ showed lower stomatal conductance for the former, due to a lower transpiration rate associated with low water bioavailability in the soil [64], which is in alignment with our overall results. Nevertheless, if we only consider ICS-95 genotype, a slight increase of stomatal conductance was observed when exposed to a higher PPFD (Table 3). Da Silva Branco et al. [10] found that a decrease in stomatal conductance in TSA-792 and TSH774 cacao genotypes was observed when plants were subjected to flooding but not when the light intensity was attenuated. These results were explained as an accumulation of abscisic acid ( $A B A)$ with the increase in shading density, regulating the stomatal opening. The opposite was observed in our study, where in TSH cacao genotypes a decrease in stomatal conductance was recorded when seedlings where exposed to a higher shade level (Table 3).

Under field conditions, Jaimez et al. [65] report that an increase in PPFD (from 400 to $1000 \mu \mathrm{mol} \mathrm{m} \mathrm{m}^{-2} \mathrm{~s}^{-1}$ ) in 7-year old cacao plot in Ecuador involved an increase in net photosynthetic rate while high stomatal conductance was maintained, even if in some cases greater stomatal conductance at a low PPFD (High shade) was recorded for some genotypes. Baligar et al. [42] reported that stomatal conductance (around $0.02 \mathrm{~mol} \mathrm{~m}^{-2}$ $\mathrm{s}^{-1}$ ) in three cacao genotypes (CCN-51, LCT EEN 37/A and VB 1117) was not significantly affected by PPFD in the range of 50 to $400 \mu \mathrm{mol} \mathrm{m} \mathrm{m}^{-2} \mathrm{~s}^{-1}$. Nonetheless, maintaining a high conductance at very low irradiance may be an advantage to understory plants, by allowing photosynthesis to respond rapidly to sunflecks.

When cacao leaves were continually exposed to light intensities higher than half of that in which instantaneous maximum photosynthesis occurred (about 350-400 $\mathrm{mmol} \mathrm{m}^{-2} \mathrm{~s}^{-1}$, which is nearly $20 \%$ of the intensity of full sunlight) the rate of photosynthesis began to decline after four hours. At light intensities higher than $100 \%$ of saturating photosynthetic intensity, the decline began almost immediately causing a certain degree of photoinhibition [38]. Taking into account these findings, our results would indicate that shade levels of $50 \%$ (PPFD of $1000 \pm 50 \mu \mathrm{mol} \mathrm{m} \mathrm{m}^{-2} \mathrm{~s}^{-1}$ ) might be too high and cause some sort of stress in terms of chlorophyll content, while the stomatal conductance increase was probably due to an increase in leaf temperature under $50 \%$ shade.

In this study, WUE was significantly affected by shade, genotypes and their interaction. WUE tended to decrease significantly with the increase of shade (Table 3 ). The average observed WUE at $50 \%$ shade was $1.32 \mathrm{~g} \mathrm{l}^{-1}$ which was significantly higher than $1.12 \mathrm{~g} \mathrm{l}^{-1}$ obtained at $80 \%$ shade. Similar tendencies were reported by Lopez-Marin et al. [66], with a negative correlation between shade levels and WUE in a greenhouse experiment with 
sweet pepper plants, registering the highest values under non-shaded conditions. Also, Yang [67] found similar tendencies in forages. Jaimez et al. [65] found in cacao growing under high and low PPFD that WUE showed a negative linear relationship with light level. and finally based on high WUE observed in the evaluated clones at $50 \%$ shade (Table 3 ), indicate the posibility of growing these cacao genotypes with less shade. On the other hand, in coffee in the dry season, Baliza et al. [68] found an increase of WUE until $50 \%$ shade and then decreases with higher levels of shade. In the current study, the maximum level of WUE $1.77 \mathrm{~g} \mathrm{l}^{-1}$ and $1.81 \mathrm{~g} \mathrm{l}^{-1}$ were recorded for ICT-1087 genotype (PFC) at $50 \%$ and $80 \%$ shade, respectively. Such high WUE in this genotype could be related to its overall highest shoot and root dry biomass at both levels of shade.

\subsection{Nutritional status influenced by shade}

Many effects of shade in cacao, are not well understood, including the differences in response to specific nutrients under shade [26]. Some cacao varieties are more nutrient demanding than others [44]. In soils with low fertility, shade acts as a buffer reducing metabolic activity which also reduces nutrient uptake. Cacao trees without shade demand higher amounts of nutrients than shaded ones: the former may contain higher levels of $\mathrm{N}$ and $\mathrm{K}$ than the latter, which had higher levels of $\mathrm{P}, \mathrm{Ca}$ and $\mathrm{Mg}$ [69].

Murray [70] reported that under shade cacao leaves had higher levels of nutrients than unshaded. In a long term study, Ahenkorah et al. [71] reported the beneficial effects of fertilization on cacao yield without shade. Macronutrients and micronutrients concentrations as influenced by genotypes and shade levels are given in Tables 4 and 5. Overall macronutrients and micronutrients concentrations were at adequate levels, however concentrations of $\mathrm{K}, \mathrm{Ca}$ and $\mathrm{Mg}$ were slightly higher and $\mathrm{P}$ concentrations were slightly lower than reported adequate levels in cacao [70-74].

In the current study, the interaction between cacao genotypes and shade levels was significant for macronutrients and micronutrients concentrations, except for $\mathrm{N}$. All macronutrients, except $\mathrm{P}$ and $\mathrm{K}$, and micronutrients concentrations were very high under $80 \%$ shade than $40 \%$ shade and significant differences were observed between two shade levels and also across genotypes. An increase of nearly $25 \%$ of the $\mathrm{N}$ concentration was recorded for cacao genotypes grown under the $80 \%$ shade level, contrary to the findings reported by Cabala Rosand et al. [69].

Overall, cacao genotypes from the four collections showed decreases in $\mathrm{Ca}$ concentration when grown under $50 \%$ shade. The highest concentrations of $P$ were 1.77 and $0.99 \mathrm{~g} \mathrm{~kg}^{-1}$ for the AYP-15 (50\% shade) and ICT-1281 (80\% shade) genotypes, respectively (Table 4 ). 
Slight differences were noted between cacao genotypes on leaf nitrogen concentration with respect to shade treatments which increased with increasing shade, contrary to $P$ which had a higher concentration under low shade. For $\mathrm{K}$ concentration, decreases in shade were associated with higher leaf $\mathrm{K}$, however, no significant differences were observed between the shade levels.

Micronutrient concentrations overall increased significantly with increasing shade from $50 \%$ to $80 \%$. Regarding micronutrients, Fe concentration at $50 \%$ shade ranged from 72 to $346 \mathrm{mg} \mathrm{kg}^{-1}$ for ICT-1189 and CP-53-C10, respectively, and from 120 to $389 \mathrm{mg} \mathrm{kg}^{-1}$ for CEPEC-2002 and UF-667 at 80\% shade, respectively. The lowest concentrations for Cu were nearly $5 \mathrm{mg} \mathrm{kg}^{-1}$ for the CP-49-C10 and UGU-112 genotypes at $50 \%$ and $80 \%$ shade, respectively. The $\mathrm{Zn}$ concentration at $50 \%$ shade ranged from 21.06 to $47.89 \mathrm{mg}$ $\mathrm{kg}^{-1}$ for IPIRANGA-1 and UNG-77, respectively, wille at $80 \%$ shade the concentration was ranged from 36.85 to $76.39 \mathrm{mg} \mathrm{kg}^{-1}$ for $\mathrm{PH}-21$ and AYP-22 respectively. Finaly the Mn concentration at $50 \%$ shade was ranged from 18.42 to $100.1 \mathrm{mg} \mathrm{kg}^{-1}$ for CA-14 and UF-667, respectively, wille at $80 \%$ shade was ranged from 50.23 to $155.58 \mathrm{mg} \mathrm{kg}^{-1}$ for UNG-53 and $\mathrm{PH}-17$, respectively. (Table 5).

Significant differences were observed in nutrient uptake between the shade levels within each cacao collection, except for Mn content (PWC, PFC and NIC) and Cu (BCC) (Figure2). K, Mg and P NUE (NIC) and Fe NUE (BCC and NIC) (Figure 3).

At $50 \%$ shade, the highest uptake of $\mathrm{K}, \mathrm{Ca}, \mathrm{Mg}, \mathrm{P}$ and $\mathrm{Zn}$ were found in $\mathrm{PWC}$, with values of $0.61,0.33,0.14,0.03 \mathrm{~g} \mathrm{plant}^{-1}$ and $0.96 \mathrm{mg} \mathrm{plant}^{-1}$, respectively; while at $80 \%$ shade, the highest uptake of $\mathrm{N}, \mathrm{K}, \mathrm{Ca}, \mathrm{Mg}, \mathrm{Cu}$ and $\mathrm{Zn}$ were found in PFC, values of $0.32 ; 0.37$, $0.26,0.11$ g plant $^{-1} ; 0.18,0.69$ mg plant $^{-1}$, respectively. The NIC at $50 \%$ and $80 \%$ shade showed the highest nutrient uptake values for Fe (4.73 and 2.98 mg plant $^{-1}$ respectively), $\mathrm{Mn}$ (1.35 and $1.23 \mathrm{mg} \mathrm{plant}^{-1}$ respectively) and $\mathrm{Cu}\left(0.25\right.$ and $0.18 \mathrm{mg} \mathrm{plant}^{-1}$ respectively) (Figure 2).

In a field study conducted in a 8-year-old cacao plantation with different shade trees and compared to a monoculture system in Ghana, Isaac et al. [75] noted that nutrient uptake by cacao increased under shade (43-80\% and $22-45 \%$ for $N$ and $P$, respectively), with $\mathrm{K}(96-140 \%)$ as the most responsive nutrient.

Baligar et al. [43] conducted a greenhouse experiment where cacao was grown at three shade levels, high, medium and low shade (PPFD of 65 $\pm 25190+46$ and 1050 $\pm 260 \mu \mathrm{mol}$ $\mathrm{m}^{-2} \mathrm{~s}^{-1}$, respectively) combined with two levels of $\mathrm{CO}_{2}\left(380\right.$ and $\left.700 \mu \mathrm{mol} \mathrm{mol}{ }^{-1}\right)$. Nutrient uptake (or content) was in the range of 158 to 168,82 to 146,40 to 86,38 to 55 , and 7.5 to $10 \mathrm{mg} \mathrm{plant}^{-1}$ for $\mathrm{N}, \mathrm{K}, \mathrm{Ca}, \mathrm{Mg}$ and $\mathrm{P}$ respectively, and in the range of 44 to 69,410 to 538, 1127 to 1764 , and 444 to $731 \mu$ plant $^{-1}$ for $\mathrm{Cu}, \mathrm{Fe}, \mathrm{Mn}$ and $\mathrm{Zn}$ respectively. At ambient $\mathrm{CO}_{2}\left(380 \mu \mathrm{mol} \mathrm{mol}{ }^{-1}\right)$, an increase of PPFD to $1050 \mu \mathrm{mol} \mathrm{m}{ }^{-2} \mathrm{~s}^{-1}$, with exception 
of $\mathrm{K}$ and $\mathrm{Mn}$ content, had a negative effect on all the essential macro and micronutrient contents. Such reduction in nutrient uptake could be attributed to a reduction in dry matter accumulation at a very high level of PPFD. 
Table 4. Mean concentrations of macronutrients $\left(\mathrm{g} \mathrm{kg}^{-1}\right)$ in shoots of 58 cacao genotypes subjected to two levels of shade $(50$ and $80 \%)$.

\begin{tabular}{|c|c|c|c|c|c|c|c|c|c|c|}
\hline \multirow{3}{*}{ Genotype } & \multicolumn{2}{|l|}{$\mathrm{N}$} & \multicolumn{2}{|l|}{$\mathrm{K}$} & \multicolumn{2}{|l|}{$\mathrm{Ca}$} & \multicolumn{2}{|l|}{$\mathrm{Mg}$} & \multicolumn{2}{|l|}{$\mathrm{P}$} \\
\hline & \multicolumn{10}{|l|}{$\mathrm{g} \mathrm{kg}^{-1}$} \\
\hline & $50 \%$ & $80 \%$ & $50 \%$ & $80 \%$ & $50 \%$ & $80 \%$ & $50 \%$ & $80 \%$ & $50 \%$ & $80 \%$ \\
\hline \multicolumn{11}{|l|}{ PWC } \\
\hline AYP-15 & $17.44 b^{*}$ & $20.77 a$ & $29.40 a$ & $23.16 \mathrm{~b}$ & $16.03 c$ & $18.51 \mathrm{~b}$ & $5.93 \mathrm{~b}$ & $6.35 b$ & $1.77 \mathrm{a}$ & $0.81 \mathrm{~d}$ \\
\hline AYP-20 & $18.45 b$ & $22.83 a$ & $30.40 a$ & $21.62 \mathrm{~b}$ & $10.67 f$ & $16.96 \mathrm{c}$ & $6.03 b$ & $6.47 \mathrm{~b}$ & $0.90 \mathrm{~d}$ & $0.69 \mathrm{e}$ \\
\hline AYP-22 & $11.10 \mathrm{~d}$ & $13.49 \mathrm{c}$ & $24.47 \mathrm{~b}$ & $22.56 \mathrm{~b}$ & $11.87 \mathrm{e}$ & $22.72 a$ & $5.03 c$ & $7.63 a$ & $1.10 \mathrm{c}$ & $0.60 \mathrm{e}$ \\
\hline PAS-100 & $19.97 a$ & $15.77 \mathrm{~b}$ & $27.23 a$ & $24.28 b$ & $17.37 \mathrm{c}$ & $18.85 b$ & $6.43 b$ & $6.57 \mathrm{~b}$ & $1.47 \mathrm{~b}$ & $0.83 d$ \\
\hline PAS-105 & $14.80 \mathrm{c}$ & $16.40 \mathrm{~b}$ & $27.93 a$ & $20.96 c$ & $16.77 \mathrm{c}$ & $17.87 \mathrm{~b}$ & $6.77 \mathrm{~b}$ & $5.92 b$ & $1.23 b$ & $0.81 \mathrm{~d}$ \\
\hline PAS-91 & $18.45 \mathrm{~b}$ & $22.83 a$ & $26.47 a$ & $20.91 \mathrm{c}$ & $12.07 \mathrm{e}$ & $16.20 c$ & $6.03 b$ & $6.20 \mathrm{~b}$ & $0.87 \mathrm{~d}$ & $0.67 \mathrm{e}$ \\
\hline PAS-93 & $17.45 \mathrm{~b}$ & $20.95 a$ & $19.67 \mathrm{c}$ & $18.39 \mathrm{c}$ & $15.70 \mathrm{c}$ & $17.23 \mathrm{c}$ & $7.17 a$ & $6.35 b$ & $1.00 \mathrm{c}$ & $0.69 \mathrm{e}$ \\
\hline UGU-112 & $10.83 d$ & $23.32 a$ & $20.90 c$ & $21.71 b$ & $9.53 f$ & $17.39 \mathrm{c}$ & $4.43 c$ & $6.20 \mathrm{~b}$ & $1.10 c$ & $0.81 \mathrm{~d}$ \\
\hline UGU-126 & $11.81 \mathrm{~d}$ & $11.09 d$ & $28.63 a$ & $22.70 \mathrm{~b}$ & $13.27 \mathrm{e}$ & $16.80 \mathrm{c}$ & $6.20 \mathrm{~b}$ & $5.74 b$ & $1.10 \mathrm{c}$ & $0.78 \mathrm{~d}$ \\
\hline UGU-130 & $11.88 \mathrm{~d}$ & $18.10 \mathrm{~b}$ & $26.00 a$ & $21.03 c$ & $16.37 \mathrm{c}$ & $15.10 \mathrm{~d}$ & $5.61 \mathrm{~b}$ & $6.60 \mathrm{~b}$ & $1.10 c$ & $0.67 \mathrm{e}$ \\
\hline UNG-53 & $17.48 \mathrm{~b}$ & $21.64 a$ & $29.90 a$ & $23.11 \mathrm{~b}$ & $16.10 c$ & $20.77 a$ & 7.30a & $7.50 a$ & $1.10 \mathrm{c}$ & $0.63 \mathrm{e}$ \\
\hline UNG-73 & $16.65 b$ & $20.60 a$ & $18.57 \mathrm{c}$ & $19.72 \mathrm{c}$ & $8.93 \mathrm{~g}$ & $14.99 \mathrm{~d}$ & $4.30 \mathrm{c}$ & $5.76 \mathrm{~b}$ & $0.72 \mathrm{~d}$ & $0.80 \mathrm{~d}$ \\
\hline UNG-76 & $10.56 \mathrm{~d}$ & $16.42 \mathrm{~b}$ & $19.80 \mathrm{c}$ & $23.19 b$ & $16.33 c$ & $15.74 \mathrm{c}$ & $6.03 \mathrm{~b}$ & $5.72 b$ & $1.33 b$ & $0.78 \mathrm{~d}$ \\
\hline UNG-77 & $17.86 \mathrm{~b}$ & $22.10 a$ & $29.60 a$ & $22.16 \mathrm{~b}$ & $12.37 \mathrm{e}$ & $15.47 \mathrm{~d}$ & $6.00 \mathrm{~b}$ & $6.59 b$ & $1.40 \mathrm{~b}$ & $0.83 d$ \\
\hline PFC & & & & & & & & & & \\
\hline ICT-1026 & $20.60 a$ & $23.06 a$ & $20.90 c$ & $23.54 b$ & $5.17 \mathrm{~h}$ & $16.95 \mathrm{c}$ & $2.23 d$ & $6.71 \mathrm{~b}$ & $1.33 b$ & $0.75 d$ \\
\hline ICT-1087 & $17.39 b$ & $21.52 \mathrm{a}$ & $19.43 c$ & $26.79 a$ & $4.43 \mathrm{~h}$ & $16.44 \mathrm{c}$ & $1.87 \mathrm{~d}$ & $6.81 \mathrm{~b}$ & $1.30 \mathrm{~b}$ & $0.83 d$ \\
\hline ICT-1092 & $14.24 \mathrm{c}$ & $17.63 \mathrm{~b}$ & $21.70 \mathrm{~b}$ & $22.14 b$ & $4.20 \mathrm{~h}$ & $17.47 \mathrm{c}$ & $2.10 \mathrm{~d}$ & $7.84 a$ & $1.17 \mathrm{c}$ & $0.77 \mathrm{~d}$ \\
\hline ICT-1112 & $18.85 b$ & $23.79 a$ & $20.17 c$ & $22.87 \mathrm{~b}$ & $4.47 \mathrm{~h}$ & $17.61 \mathrm{c}$ & $1.97 \mathrm{~d}$ & $8.65 a$ & $1.43 b$ & $0.78 \mathrm{~d}$ \\
\hline ICT-1189 & $18.03 \mathrm{~b}$ & $22.31 a$ & $18.83 c$ & $24.27 \mathrm{~b}$ & $14.87 \mathrm{~d}$ & $16.59 \mathrm{c}$ & $5.50 \mathrm{~b}$ & $6.21 \mathrm{~b}$ & $1.27 \mathrm{~b}$ & $0.84 d$ \\
\hline ICT-1251 & $17.46 \mathrm{~b}$ & $21.61 a$ & $21.50 \mathrm{~b}$ & $26.06 a$ & $5.00 \mathrm{~h}$ & $18.81 b$ & $2.23 \mathrm{~d}$ & $6.25 b$ & $1.10 \mathrm{c}$ & $0.75 d$ \\
\hline ICT-1281 & $18.86 \mathrm{~b}$ & $27.12 a$ & $19.83 c$ & $24.87 \mathrm{~b}$ & $13.17 \mathrm{e}$ & $16.67 \mathrm{c}$ & $6.07 \mathrm{~b}$ & $6.69 b$ & $1.00 c$ & $0.99 \mathrm{c}$ \\
\hline ICT-1292 & $18.62 b$ & $23.05 a$ & $21.50 \mathrm{~b}$ & $26.89 a$ & $4.87 \mathrm{~h}$ & $18.89 \mathrm{~b}$ & $2.43 d$ & $7.70 a$ & $1.30 \mathrm{~b}$ & $0.84 d$ \\
\hline ICT-1506 & $14.55 c$ & $18.01 \mathrm{~b}$ & $21.87 \mathrm{~b}$ & $26.66 a$ & $4.47 \mathrm{~h}$ & $18.45 b$ & $2.07 \mathrm{~d}$ & $7.43 a$ & $1.17 \mathrm{c}$ & $0.88 \mathrm{~d}$ \\
\hline ICT-2142 & $18.18 \mathrm{~b}$ & $22.35 a$ & $21.90 \mathrm{~b}$ & $22.37 \mathrm{~b}$ & $12.87 \mathrm{e}$ & $17.13 \mathrm{c}$ & $6.77 \mathrm{~b}$ & $6.76 \mathrm{~b}$ & $1.10 \mathrm{c}$ & $0.81 \mathrm{~d}$ \\
\hline ICT-2161 & $16.53 b$ & $20.46 a$ & $21.47 \mathrm{~b}$ & $20.87 c$ & $12.10 \mathrm{e}$ & $16.01 \mathrm{c}$ & $6.23 b$ & $7.10 a$ & $0.77 \mathrm{~d}$ & $0.57 \mathrm{e}$ \\
\hline ICT-2171 & $18.76 \mathrm{~b}$ & $23.57 a$ & $21.10 \mathrm{c}$ & $23.97 \mathrm{~b}$ & $4.77 \mathrm{~h}$ & $19.61 b$ & $2.17 \mathrm{~d}$ & $8.23 a$ & $1.18 \mathrm{c}$ & $0.81 \mathrm{~d}$ \\
\hline ICT-2172 & $17.41 \mathrm{~b}$ & $22.30 a$ & $23.57 \mathrm{~b}$ & $27.92 a$ & $5.13 \mathrm{~h}$ & $18.77 \mathrm{~b}$ & $2.33 d$ & $7.58 a$ & $1.37 \mathrm{~b}$ & $0.77 \mathrm{~d}$ \\
\hline ICT-2173 & $19.40 \mathrm{~b}$ & $24.00 a$ & $22.03 b$ & $26.74 a$ & $14.03 d$ & $16.42 \mathrm{c}$ & $6.47 \mathrm{~b}$ & $7.10 a$ & $1.30 \mathrm{~b}$ & $0.96 c$ \\
\hline $\begin{array}{l}\text { ICT-2653 } \\
\text { BCC }\end{array}$ & $18.77 \mathrm{~b}$ & $23.22 a$ & $21.73 b$ & $23.96 \mathrm{~b}$ & $7.60 \mathrm{~g}$ & $19.28 b$ & $2.00 \mathrm{~d}$ & $6.90 a$ & $0.83 d$ & $0.61 \mathrm{e}$ \\
\hline$\overline{\mathrm{BN}-34}$ & $22.59 a$ & $23.83 a$ & $22.10 \mathrm{~b}$ & $24.55 b$ & $12.70 \mathrm{e}$ & $19.1 \mathrm{~b}$ & $5.70 \mathrm{~b}$ & $7.41 a$ & $1.03 c$ & $0.68 \mathrm{e}$ \\
\hline BS-01 & $18.86 \mathrm{~b}$ & $22.92 a$ & $22.70 \mathrm{~b}$ & $20.12 c$ & $15.23 d$ & $19.65 b$ & $6.23 b$ & $9.72 a$ & $0.97 \mathrm{c}$ & $0.30 \mathrm{e}$ \\
\hline CA-14 & $17.86 \mathrm{~b}$ & $22.11 a$ & $23.17 \mathrm{~b}$ & $24.34 b$ & $13.10 \mathrm{e}$ & $15.88 \mathrm{c}$ & $5.27 \mathrm{~b}$ & $6.87 a$ & $1.00 \mathrm{c}$ & $0.69 \mathrm{e}$ \\
\hline CEPEC-2002 & $18.38 \mathrm{~b}$ & $22.12 a$ & $26.07 a$ & $23.59 \mathrm{~b}$ & $11.77 \mathrm{e}$ & $22.82 a$ & $5.43 b$ & $8.12 a$ & $1.07 \mathrm{c}$ & $0.53 \mathrm{e}$ \\
\hline CP-49-C10 & $18.86 \mathrm{~b}$ & $23.29 a$ & $21.53 b$ & $20.78 c$ & $12.90 \mathrm{e}$ & $18.92 b$ & $5.77 \mathrm{~b}$ & $8.03 a$ & $0.77 \mathrm{~d}$ & $0.49 \mathrm{e}$ \\
\hline CP-53-C10 & $19.72 a$ & $24.40 a$ & $21.63 b$ & $25.03 b$ & $12.53 \mathrm{e}$ & $18.85 \mathrm{~b}$ & $5.60 \mathrm{~b}$ & $7.74 a$ & $0.97 \mathrm{c}$ & $0.60 \mathrm{e}$ \\
\hline IPIRANGA-1 & $17.18 \mathrm{~b}$ & $21.26 a$ & $22.37 \mathrm{~b}$ & $22.59 \mathrm{~b}$ & $10.70 f$ & $16.49 \mathrm{c}$ & $5.40 \mathrm{~b}$ & $6.94 a$ & $0.94 \mathrm{~d}$ & $0.42 \mathrm{e}$ \\
\hline PH-09 & $17.94 \mathrm{~b}$ & $21.95 a$ & $22.37 \mathrm{~b}$ & $19.06 \mathrm{c}$ & $11.33 \mathrm{f}$ & $20.99 a$ & $5.67 \mathrm{~b}$ & $7.47 a$ & $1.08 \mathrm{c}$ & $0.53 \mathrm{e}$ \\
\hline PH-144 & $20.06 a$ & $24.83 a$ & $21.97 \mathrm{~b}$ & $20.57 \mathrm{c}$ & $13.27 \mathrm{e}$ & $18.32 \mathrm{~b}$ & $5.83 b$ & $7.34 a$ & $0.97 c$ & $0.60 \mathrm{e}$ \\
\hline PH-15 & $17.97 \mathrm{~b}$ & $22.72 a$ & $26.05 a$ & $21.33 b$ & $12.75 \mathrm{e}$ & $17.57 \mathrm{c}$ & $5.75 b$ & $7.12 a$ & $0.95 \mathrm{c}$ & $0.62 \mathrm{e}$ \\
\hline PH-16 & $18.45 b$ & $22.83 a$ & $20.53 c$ & $19.10 c$ & $14.40 \mathrm{~d}$ & $21.81 \mathrm{a}$ & $6.30 \mathrm{~b}$ & $7.70 a$ & $1.30 \mathrm{~b}$ & $0.50 \mathrm{e}$ \\
\hline PH-17 & $17.22 \mathrm{~b}$ & $21.28 a$ & $22.67 \mathrm{~b}$ & $19.54 \mathrm{c}$ & $13.87 \mathrm{~d}$ & $18.77 \mathrm{~b}$ & $5.77 \mathrm{~b}$ & $7.42 a$ & $0.70 \mathrm{~d}$ & $0.48 \mathrm{e}$ \\
\hline PH-21 & $18.28 b$ & $23.93 a$ & $23.97 \mathrm{~b}$ & $17.54 \mathrm{c}$ & $11.70 \mathrm{e}$ & $19.16 \mathrm{~b}$ & $5.57 \mathrm{~b}$ & $7.74 a$ & $0.93 d$ & $0.55 \mathrm{e}$ \\
\hline $\begin{array}{l}\text { PH-990 } \\
\text { NIC }\end{array}$ & $18.35 b$ & $28.83 a$ & $24.87 \mathrm{~b}$ & $23.12 b$ & $10.93 f$ & $18.73 b$ & $4.46 c$ & $7.72 a$ & $0.83 d$ & $0.63 \mathrm{e}$ \\
\hline $\mathrm{CCN}-10$ & $17.63 \mathrm{~b}$ & $21.81 \mathrm{a}$ & $12.37 \mathrm{~d}$ & $17.19 \mathrm{c}$ & $7.23 \mathrm{~g}$ & $14.28 d$ & $4.03 c$ & $4.69 c$ & $0.63 \mathrm{e}$ & $0.84 d$ \\
\hline CCN-51 & $18.73 b$ & $23.18 a$ & $13.87 \mathrm{~d}$ & $17.81 \mathrm{c}$ & $8.33 \mathrm{~g}$ & $12.53 \mathrm{e}$ & $3.80 c$ & $4.92 \mathrm{c}$ & $1.10 \mathrm{c}$ & $0.80 \mathrm{~d}$ \\
\hline EET-400 & $17.64 \mathrm{~b}$ & $21.83 a$ & $24.93 b$ & $19.81 \mathrm{c}$ & $15.13 d$ & $18.46 \mathrm{~b}$ & 7.93a & $6.60 \mathrm{~b}$ & $0.93 d$ & $0.78 \mathrm{~d}$ \\
\hline $\mathrm{H}-10$ & $14.97 \mathrm{c}$ & $18.52 \mathrm{~b}$ & $12.20 \mathrm{~d}$ & $18.98 \mathrm{c}$ & $6.70 \mathrm{~g}$ & $17.51 \mathrm{c}$ & $3.70 c$ & $6.42 \mathrm{~b}$ & $0.25 \mathrm{e}$ & $0.62 \mathrm{e}$ \\
\hline ICS-1 & $18.04 \mathrm{~b}$ & $26.54 a$ & $13.43 d$ & $16.44 d$ & $7.57 \mathrm{~g}$ & $12.36 \mathrm{e}$ & $3.50 c$ & $5.72 b$ & $1.10 \mathrm{c}$ & $0.89 \mathrm{~d}$ \\
\hline ICS-39 & $17.24 b$ & $21.33 a$ & $17.70 \mathrm{c}$ & $19.68 \mathrm{c}$ & $10.47 f$ & $15.39 d$ & $4.06 c$ & $6.18 b$ & $0.63 e$ & $0.91 d$ \\
\hline ICS-6 & $15.22 \mathrm{c}$ & $21.20 \mathrm{a}$ & $26.03 a$ & $21.18 \mathrm{c}$ & $13.23 \mathrm{e}$ & $15.75 \mathrm{c}$ & $7.43 a$ & $6.35 b$ & $0.97 c$ & $0.83 d$ \\
\hline ICS-95 & $17.54 \mathrm{~b}$ & $21.71 a$ & $24.13 b$ & $18.97 \mathrm{c}$ & $13.43 \mathrm{e}$ & $13.72 \mathrm{~d}$ & $7.13 a$ & $4.66 \mathrm{c}$ & $0.93 d$ & $0.90 \mathrm{~d}$ \\
\hline IMC-67 & $18.26 \mathrm{~b}$ & $22.60 a$ & $16.46 \mathrm{~d}$ & $22.13 b$ & $9.33 \mathrm{f}$ & $17.68 \mathrm{~b}$ & $5.42 \mathrm{~b}$ & $6.95 a$ & $1.45 b$ & $0.72 d$ \\
\hline POUND-12 & $17.60 \mathrm{~b}$ & $21.82 a$ & $22.35 b$ & $19.63 c$ & $14.35 \mathrm{~d}$ & $14.88 \mathrm{~d}$ & $6.70 \mathrm{~b}$ & $5.97 \mathrm{~b}$ & $0.80 \mathrm{~d}$ & $0.84 d$ \\
\hline SCA-6 & $16.17 \mathrm{~b}$ & $20.01 a$ & $13.17 \mathrm{~d}$ & $18.25 \mathrm{c}$ & $8.43 \mathrm{~g}$ & $16.87 \mathrm{c}$ & $4.87 \mathrm{c}$ & $6.37 \mathrm{~b}$ & $0.60 \mathrm{e}$ & $0.72 d$ \\
\hline TSH-1188 & $17.27 \mathrm{~b}$ & $21.37 a$ & $24.70 \mathrm{~b}$ & $14.72 \mathrm{~d}$ & $12.9 \mathrm{e}$ & $11.86 \mathrm{e}$ & $7.33 a$ & $4.64 \mathrm{c}$ & $0.87 \mathrm{~d}$ & $0.89 \mathrm{~d}$ \\
\hline TSH-565 & $16.83 \mathrm{~b}$ & $20.82 a$ & $23.40 \mathrm{~b}$ & $16.27 d$ & $14.70 \mathrm{~d}$ & $14.66 \mathrm{~d}$ & $7.73 a$ & $5.11 \mathrm{~b}$ & $0.97 c$ & $0.77 \mathrm{~d}$ \\
\hline UF-613 & $18.26 \mathrm{~b}$ & $22.59 a$ & $13.60 \mathrm{~d}$ & $17.22 \mathrm{c}$ & $9.20 f$ & $16.55 \mathrm{c}$ & $4.20 c$ & $6.39 b$ & $1.20 \mathrm{c}$ & $0.98 \mathrm{c}$ \\
\hline UF-667 & $16.64 b$ & $20.61 a$ & $23.53 b$ & $19.73 c$ & $14.10 \mathrm{~d}$ & $14.67 \mathrm{~d}$ & $7.90 \mathrm{a}$ & $5.61 \mathrm{~b}$ & $0.83 \mathrm{~d}$ & $0.96 c$ \\
\hline Media PWC & $15.09 d$ & $19.02 b$ & $25.64 a$ & $21.82 \mathrm{c}$ & $13.81 \mathrm{~d}$ & $17.47 \mathrm{~b}$ & $6.02 \mathrm{c}$ & $6.40 \mathrm{~b}$ & $1.18 a$ & $0.74 \mathrm{c}$ \\
\hline Media PFC & $17.45 \mathrm{c}$ & $21.78 a$ & $21.17 \mathrm{c}$ & $24.66 a$ & $7.81 \mathrm{f}$ & $17.67 \mathrm{~b}$ & $3.60 \mathrm{~d}$ & $7.20 a$ & $1.14 a$ & $0.80 c$ \\
\hline Media BCC & $18.26 \mathrm{~b}$ & $22.53 a$ & $23.00 \mathrm{~b}$ & $21.52 \mathrm{c}$ & $12.66 \mathrm{~d}$ & $19.08 a$ & $5.69 c$ & $7.52 a$ & $0.93 b$ & $0.54 d$ \\
\hline Media NIC & $17.20 \mathrm{c}$ & $21.45 a$ & $18.79 \mathrm{~d}$ & $18.53 d$ & $11.01 \mathrm{e}$ & $15.14 \mathrm{c}$ & $5.78 \mathrm{c}$ & $5.69 c$ & $0.87 \mathrm{~b}$ & $0.83 c$ \\
\hline Media Total & $17.01 \mathrm{~B}^{\star \star}$ & $21.21 \mathrm{~A}$ & $22.08 B$ & $21.63 \mathrm{~A}$ & $11.26 \mathrm{~B}$ & $17.31 \mathrm{~A}$ & $5.25 \mathrm{~B}$ & $6.69 \mathrm{~A}$ & $1.03 \mathrm{~A}$ & $0.73 B$ \\
\hline $\begin{array}{c}\text { Source of } \\
\text { variation }\end{array}$ & & & & & & & & & & \\
\hline Shade (S) & $<0.0001$ & & 0.1034 & & $<0.0001$ & & $<0.0001$ & & 0.0002 & \\
\hline Genotype (G) 57 & $<0.0001$ & & $<0.0001$ & & $<0.0001$ & & $<0.0001$ & & $<0.0001$ & \\
\hline$G^{*} \mathrm{~S}$ & 0.0022 & & $<0.0001$ & & $<0.0001$ & & $<0.0001$ & & $<0.0001$ & \\
\hline
\end{tabular}

$\left({ }^{*}\right)$ Different lower case letters on the right of each value in both columns of each variable indicate a significant difference between genotypes and shade level, (Scott \& Knott test, $(P<0.05)$. $\left(^{* *}\right)$ Different capital letters on the right of each value in line of each variable indicate a significant difference between shade level (Scott \& Knott test, $(P<0.05)$. PWC: wild cacao from river basins of Peruvian Amazon collection, PFC: Peruvian farmers' collection, BCC: Brazilian cacao collection, NIC: National and International cacao collection. Different lower case letters in parenthesis on the right of each value in both columns of each variable indicate a significant difference between genotypes and shade level, (Scott \& Knott test, $(P<0.05)$. 
Table 5. Mean concentration of micronutrients $\left(\mathrm{mg} \mathrm{kg}^{-1}\right)$ in shoots of 58 cacao genotypes subjected to two levels of shade(50 and $80 \%)$.

\begin{tabular}{|c|c|c|c|c|c|c|c|c|}
\hline \multirow{3}{*}{ Genotype } & \multirow{2}{*}{\multicolumn{2}{|c|}{$\begin{array}{l}\mathrm{Fe} \\
\mathrm{mg} \mathrm{kg}^{-1}\end{array}$}} & \multicolumn{2}{|l|}{$\mathrm{Zn}$} & \multicolumn{2}{|l|}{$\mathrm{Mn}$} & \multicolumn{2}{|l|}{$\mathrm{Cu}$} \\
\hline & & & & & & & & \\
\hline & $50 \%$ & $80 \%$ & $50 \%$ & $80 \%$ & $50 \%$ & $80 \%$ & $50 \%$ & $80 \%$ \\
\hline \multicolumn{9}{|l|}{ PWC } \\
\hline$\overline{\mathrm{AYP}}-15$ & $247.80 b^{*}$ & $259.18 b$ & $46.57 \mathrm{~b}$ & $42.23 c$ & $84.29 b$ & $63.46 c$ & $4.42 \mathrm{c}$ & $8.89 c$ \\
\hline AYP-20 & $169.84 c$ & $278.63 a$ & $31.88 \mathrm{~d}$ & $45.01 \mathrm{c}$ & $54.25 \mathrm{c}$ & $84.93 b$ & $6.74 \mathrm{c}$ & $9.35 \mathrm{c}$ \\
\hline AYP-22 & $187.13 \mathrm{c}$ & $222.38 b$ & $33.66 \mathrm{~d}$ & $76.39 a$ & $28.37 d$ & $84.18 b$ & $6.88 \mathrm{c}$ & $16.50 \mathrm{~b}$ \\
\hline PAS-100 & $275.73 a$ & $225.13 b$ & $43.36 c$ & $50.00 \mathrm{~b}$ & $70.38 \mathrm{~b}$ & $100.18 b$ & $9.54 \mathrm{c}$ & $7.50 \mathrm{c}$ \\
\hline PAS-105 & $151.18 \mathrm{c}$ & $239.34 b$ & $43.24 c$ & $55.80 \mathrm{~b}$ & $49.54 c$ & 131.91a & $8.09 c$ & $12.92 \mathrm{~b}$ \\
\hline PAS-91 & $149.98 \mathrm{c}$ & $225.64 b$ & $41.47 \mathrm{c}$ & $47.31 \mathrm{~b}$ & $53.07 \mathrm{c}$ & $115.15 a$ & $5.09 c$ & $6.54 \mathrm{c}$ \\
\hline PAS-93 & $122.26 \mathrm{~d}$ & $225.84 b$ & $38.96 c$ & $47.19 b$ & $65.03 c$ & $75.48 \mathrm{~b}$ & $6.65 \mathrm{c}$ & $14.33 \mathrm{~b}$ \\
\hline UGU-112 & $131.94 d$ & $226.99 b$ & $32.82 \mathrm{~d}$ & $50.12 b$ & $22.63 d$ & $64.79 c$ & $6.01 \mathrm{c}$ & $4.92 \mathrm{c}$ \\
\hline UGU-126 & $168.27 c$ & $245.70 \mathrm{~b}$ & $39.73 c$ & $42.50 c$ & $46.85 \mathrm{c}$ & $80.83 b$ & $5.17 \mathrm{c}$ & $6.78 \mathrm{c}$ \\
\hline UGU-130 & $166.49 \mathrm{c}$ & $225.14 b$ & $47.72 b$ & $47.03 b$ & $55.25 \mathrm{c}$ & $78.62 b$ & $7.77 \mathrm{c}$ & $6.47 \mathrm{c}$ \\
\hline UNG-53 & $177.41 \mathrm{c}$ & $182.02 \mathrm{c}$ & $44.73 c$ & $42.59 c$ & $58.87 \mathrm{c}$ & $50.23 c$ & $9.44 c$ & $9.13 c$ \\
\hline UNG-73 & $107.39 d$ & $260.63 b$ & $31.31 d$ & $41.17 \mathrm{c}$ & $18.52 \mathrm{~d}$ & $79.68 b$ & $6.84 c$ & $7.77 \mathrm{c}$ \\
\hline UNG-76 & $120.68 d$ & $303.51 a$ & $42.18 c$ & $41.53 c$ & $40.11 d$ & $77.83 \mathrm{~b}$ & $8.06 \mathrm{c}$ & $5.95 \mathrm{c}$ \\
\hline \multicolumn{8}{|l|}{ PFC } & $14.99 \mathrm{~b}$ \\
\hline ICT-1026 & $98.48 d$ & $176.44 \mathrm{c}$ & $35.25 d$ & $48.75 b$ & $58.83 c$ & $50.67 c$ & $9.66 c$ & $24.26 a$ \\
\hline ICT-1087 & $109.82 d$ & $173.16 \mathrm{c}$ & $26.16 \mathrm{~d}$ & $41.61 \mathrm{c}$ & $47.81 \mathrm{c}$ & $82.21 b$ & $9.80 \mathrm{c}$ & $16.01 \mathrm{~b}$ \\
\hline ICT-1092 & $80.33 d$ & $185.94 \mathrm{c}$ & $30.58 d$ & $50.88 b$ & $50.24 c$ & $105.24 a$ & $5.87 \mathrm{c}$ & $10.70 c$ \\
\hline ICT-1112 & $84.37 d$ & $141.48 d$ & $40.87 \mathrm{c}$ & $44.44 \mathrm{c}$ & $28.94 d$ & $54.33 \mathrm{c}$ & $6.64 \mathrm{c}$ & $8.38 \mathrm{c}$ \\
\hline ICT-1189 & $72.04 d$ & $142.24 d$ & $26.86 \mathrm{~d}$ & $42.41 \mathrm{c}$ & $52.35 \mathrm{c}$ & $62.81 \mathrm{c}$ & $5.32 \mathrm{c}$ & $8.69 \mathrm{c}$ \\
\hline ICT-1251 & $177.47 \mathrm{c}$ & $135.31 d$ & $45.55 c$ & $51.08 b$ & $63.69 c$ & $92.37 b$ & $6.56 \mathrm{c}$ & $8.21 \mathrm{c}$ \\
\hline ICT-1281 & $109.77 d$ & $134.68 d$ & $29.39 d$ & $45.75 c$ & $42.80 \mathrm{c}$ & $67.15 c$ & $5.02 \mathrm{c}$ & $10.97 c$ \\
\hline ICT-1292 & $127.43 d$ & $155.29 \mathrm{c}$ & $30.19 d$ & $47.28 b$ & $43.96 c$ & $62.06 c$ & $6.75 c$ & $16.72 b$ \\
\hline ICT-1506 & $133.08 d$ & $151.98 \mathrm{c}$ & $37.90 c$ & $44.51 \mathrm{c}$ & $40.41 d$ & $74.93 \mathrm{~b}$ & $7.90 \mathrm{c}$ & $9.44 \mathrm{c}$ \\
\hline ICT-2142 & $127.25 d$ & $156.95 \mathrm{c}$ & $25.04 d$ & $39.70 c$ & $39.12 d$ & $50.95 c$ & $7.61 \mathrm{c}$ & $16.54 \mathrm{~b}$ \\
\hline ICT-2161 & $112.34 d$ & $138.54 d$ & $22.86 \mathrm{~d}$ & $46.56 \mathrm{~b}$ & $31.96 \mathrm{~d}$ & $81.67 \mathrm{~b}$ & $9.06 \mathrm{c}$ & $9.38 \mathrm{c}$ \\
\hline ICT-2171 & $123.40 \mathrm{~d}$ & $188.00 \mathrm{c}$ & $42.32 c$ & $50.65 b$ & $45.54 c$ & $81.72 b$ & $8.18 \mathrm{c}$ & $19.50 \mathrm{~b}$ \\
\hline ICT-2172 & $92.32 \mathrm{~d}$ & $162.03 c$ & $33.01 \mathrm{~d}$ & $45.42 c$ & $36.20 \mathrm{~d}$ & $70.56 b$ & $8.39 c$ & $9.03 c$ \\
\hline ICT-2173 & $110.90 \mathrm{~d}$ & $131.38 d$ & $27.19 d$ & $48.07 \mathrm{~b}$ & $47.83 c$ & $74.68 \mathrm{~b}$ & $8.72 \mathrm{c}$ & $9.02 \mathrm{c}$ \\
\hline \multicolumn{9}{|l|}{ BCC } \\
\hline$\overline{\mathrm{BN}-34}$ & $108.44 d$ & $207.65 b$ & $29.92 d$ & $38.60 c$ & $31.76 \mathrm{~d}$ & $72.46 \mathrm{~b}$ & $5.19 c$ & $9.42 \mathrm{c}$ \\
\hline BS-01 & $258.90 \mathrm{~b}$ & $205.16 \mathrm{~b}$ & $24.13 d$ & $40.69 c$ & $34.11 \mathrm{~d}$ & $57.58 c$ & $4.41 \mathrm{c}$ & $5.84 c$ \\
\hline CA-14 & $126.32 d$ & $155.77 \mathrm{c}$ & $26.11 d$ & $38.29 c$ & $18.42 d$ & $52.78 c$ & $5.17 \mathrm{c}$ & $7.01 \mathrm{c}$ \\
\hline CEPEC-2002 & $159.11 \mathrm{c}$ & $119.81 d$ & $38.74 \mathrm{c}$ & $49.70 b$ & $21.80 \mathrm{~d}$ & $34.36 \mathrm{~d}$ & $6.04 c$ & $9.20 \mathrm{c}$ \\
\hline CP-49-C10 & $97.20 \mathrm{~d}$ & $141.37 d$ & $22.02 \mathrm{~d}$ & $48.66 \mathrm{~b}$ & $33.31 \mathrm{~d}$ & $38.69 d$ & $4.13 c$ & $7.74 \mathrm{c}$ \\
\hline CP-53-C10 & $345.71 a$ & $179.01 \mathrm{c}$ & $21.64 d$ & $46.34 b$ & $38.32 d$ & $78.28 b$ & $5.72 \mathrm{c}$ & $9.93 c$ \\
\hline IPIRANGA-1 & $121.31 d$ & $234.17 \mathrm{~b}$ & $21.06 \mathrm{~d}$ & $44.22 c$ & $25.86 \mathrm{~d}$ & $95.32 b$ & $5.45 c$ & $7.17 \mathrm{c}$ \\
\hline PH-09 & $213.20 \mathrm{~b}$ & $171.92 \mathrm{c}$ & $25.17 d$ & $43.18 c$ & $26.35 d$ & $122.81 a$ & $6.11 \mathrm{c}$ & $9.18 \mathrm{c}$ \\
\hline PH-144 & $243.08 b$ & $189.39 c$ & $23.46 \mathrm{~d}$ & $41.19 c$ & $32.13 d$ & $56.63 c$ & $4.48 \mathrm{c}$ & $5.20 c$ \\
\hline PH-15 & $116.92 d$ & $198.69 \mathrm{~b}$ & $27.91 d$ & $40.08 c$ & $21.31 d$ & $48.97 \mathrm{c}$ & $6.78 \mathrm{c}$ & $7.81 \mathrm{c}$ \\
\hline PH-16 & $329.32 a$ & $211.58 \mathrm{~b}$ & $21.59 d$ & $53.52 b$ & $30.70 \mathrm{~d}$ & $147.17 a$ & $6.02 \mathrm{c}$ & $7.29 \mathrm{c}$ \\
\hline $\mathrm{PH}-17$ & $135.13 d$ & $156.04 \mathrm{c}$ & $34.53 d$ & $60.42 b$ & $49.64 c$ & $155.58 a$ & $6.77 \mathrm{c}$ & $13.69 \mathrm{~b}$ \\
\hline $\mathrm{PH}-21$ & $194.97 \mathrm{~b}$ & $200.87 \mathrm{~b}$ & $29.07 d$ & $36.85 c$ & $22.05 \mathrm{~d}$ & $52.47 \mathrm{c}$ & $6.30 \mathrm{c}$ & $6.82 \mathrm{c}$ \\
\hline PH-990 & $274.37 a$ & $167.23 \mathrm{c}$ & $43.07 \mathrm{c}$ & $41.27 \mathrm{c}$ & $24.12 d$ & $64.56 \mathrm{c}$ & $7.39 c$ & $10.27 \mathrm{c}$ \\
\hline \multicolumn{9}{|c|}{$901.200 \quad+0.010$} \\
\hline$\overline{\mathrm{CCN}}-10$ & $262.12 \mathrm{~b}$ & $205.56 \mathrm{~b}$ & $43.74 c$ & $43.83 c$ & $84.95 \mathrm{~b}$ & $101.23 \mathrm{~b}$ & $9.07 \mathrm{c}$ & $12.96 \mathrm{~b}$ \\
\hline CCN-51 & $261.34 b$ & $230.53 b$ & $26.89 d$ & $37.04 \mathrm{c}$ & $21.36 \mathrm{~d}$ & $88.85 b$ & $9.78 c$ & $12.86 \mathrm{~b}$ \\
\hline EET-400 & $239.43 \mathrm{~b}$ & $256.67 \mathrm{~b}$ & $44.05 c$ & $55.18 b$ & $82.36 \mathrm{~b}$ & $73.81 \mathrm{~b}$ & $15.63 b$ & $15.86 \mathrm{~b}$ \\
\hline $\mathrm{H}-10$ & $122.26 \mathrm{~d}$ & $152.02 \mathrm{c}$ & $44.78 c$ & $39.49 c$ & $27.82 d$ & $65.85 c$ & $8.38 \mathrm{c}$ & $17.54 \mathrm{~b}$ \\
\hline ICS-1 & $199.54 b$ & $183.69 \mathrm{c}$ & $34.11 d$ & $37.63 c$ & $21.10 \mathrm{~d}$ & $103.17 \mathrm{~b}$ & $9.71 \mathrm{c}$ & $11.77 \mathrm{c}$ \\
\hline ICS-39 & $140.60 \mathrm{~d}$ & $167.99 \mathrm{c}$ & $45.35 c$ & $45.45 c$ & $74.68 \mathrm{~b}$ & $109.90 a$ & $10.9 \mathrm{c}$ & $9.02 \mathrm{c}$ \\
\hline ICS-6 & $165.72 \mathrm{c}$ & $206.67 \mathrm{~b}$ & $36.59 c$ & $40.83 c$ & $55.47 \mathrm{c}$ & $75.83 \mathrm{~b}$ & $12.58 \mathrm{~b}$ & $15.83 \mathrm{~b}$ \\
\hline ICS-95 & $198.72 b$ & $205.77 \mathrm{~b}$ & $41.87 \mathrm{c}$ & $42.48 \mathrm{c}$ & $78.05 \mathrm{~b}$ & $126.84 a$ & $18.79 b$ & $29.92 a$ \\
\hline IMC-67 & $247.17 \mathrm{~b}$ & $203.83 b$ & $38.20 c$ & $44.21 \mathrm{c}$ & $26.75 d$ & $79.31 b$ & $9.43 c$ & $12.93 \mathrm{~b}$ \\
\hline POUND-12 & $184.32 \mathrm{c}$ & $183.17 \mathrm{c}$ & $41.13 c$ & $41.83 c$ & $59.54 c$ & $64.34 \mathrm{c}$ & $7.64 c$ & $7.23 \mathrm{c}$ \\
\hline SCA-6 & $134.50 \mathrm{~d}$ & $219.24 b$ & $40.45 c$ & $44.61 \mathrm{c}$ & $70.05 b$ & $66.24 c$ & $11.68 \mathrm{c}$ & $20.08 \mathrm{~b}$ \\
\hline TSH-1188 & $189.65 \mathrm{c}$ & $226.59 b$ & $38.94 \mathrm{c}$ & $46.50 \mathrm{~b}$ & $54.22 \mathrm{c}$ & $115.98 a$ & $7.16 c$ & $8.87 \mathrm{c}$ \\
\hline TSH-565 & $236.09 \mathrm{~b}$ & $213.47 \mathrm{~b}$ & $36.34 \mathrm{c}$ & $47.32 \mathrm{~b}$ & $83.48 \mathrm{~b}$ & $76.51 \mathrm{~b}$ & $8.08 \mathrm{c}$ & $12.44 \mathrm{~b}$ \\
\hline UF-613 & $174.97 \mathrm{c}$ & $229.17 b$ & $37.61 \mathrm{c}$ & $43.42 \mathrm{c}$ & $18.86 \mathrm{~d}$ & $93.03 \mathrm{~b}$ & $14.56 \mathrm{~b}$ & $14.16 \mathrm{~b}$ \\
\hline UF-667 & $286.85 a$ & $388.64 a$ & $38.00 \mathrm{c}$ & $37.94 \mathrm{c}$ & $100.10 \mathrm{~b}$ & $82.76 \mathrm{~b}$ & $9.44 \mathrm{c}$ & $10.78 \mathrm{c}$ \\
\hline Media PWC & $173.09 \mathrm{~b}$ & $240.11 a$ & $40.39 c$ & $47.91 a$ & $50.60 \mathrm{~b}$ & $84.15 a$ & $7.04 d$ & $9.43 c$ \\
\hline Media PFC & $110.65 d$ & $156.13 c$ & $32.26 \mathrm{~d}$ & $46.62 a$ & $44.73 b$ & $72.87 a$ & $7.49 \mathrm{~d}$ & $12.42 \mathrm{~b}$ \\
\hline Media BCC & $194.57 b$ & $181.33 \mathrm{~b}$ & $27.74 \mathrm{e}$ & $44.50 \mathrm{~b}$ & $29.28 c$ & $76.98 a$ & $5.71 \mathrm{~d}$ & $8.33 \mathrm{c}$ \\
\hline Media NIC & $202.88 b$ & $218.20 a$ & $39.20 \mathrm{c}$ & $43.18 b$ & $57.25 b$ & $88.24 a$ & $10.85 b$ & $14.15 a$ \\
\hline Media Total & $169.83 B$ & $198.54 \mathrm{~A}$ & 34.93B & $45.53 \mathrm{~A}$ & $45.66 \mathrm{~B}$ & $80.56 \mathrm{~A}$ & $7.82 \mathrm{~B}$ & $11.16 \mathrm{~A}$ \\
\hline $\begin{array}{l}\text { Source of } \\
\text { variation }\end{array}$ & $p v$ & & & & & & & \\
\hline $\begin{array}{ll}\text { Shade }(\mathrm{S}) & 1\end{array}$ & 0.0017 & & $<0.0001$ & & 0.0006 & & $<0.0001$ & \\
\hline Genotype (G) 57 & $<0.0001$ & & $<0.0001$ & & $<0.0001$ & & $<0.0001$ & \\
\hline$G * S$ & $<0.0001$ & & $<0.0001$ & & $<0.0001$ & & 0.0017 & \\
\hline
\end{tabular}

$\left({ }^{*}\right)$ Different lower case letters on the right of each value in both columns of each variable indicate a significant difference between genotypes and shade level, (Scott \& Knott test, $(P<0.05)$. ( $\left.{ }^{* *}\right)$ Different capital letters on the right of each value in line of each variable indicate a significant difference between shade level (Scott \& Knott test, $(P<0.05)$. PWC: wild cacao from river basins of Peruvian Amazon collection, PFC: Peruvian farmers' collection, BCC: Brazilian cacao collection, NIC: National and International cacao collection. Different lower case letters in parenthesis on the right of each value in both columns of each variable indicate a significant difference between genotypes and shade level, (Scott \& Knott test, $(P<0.05)$. 

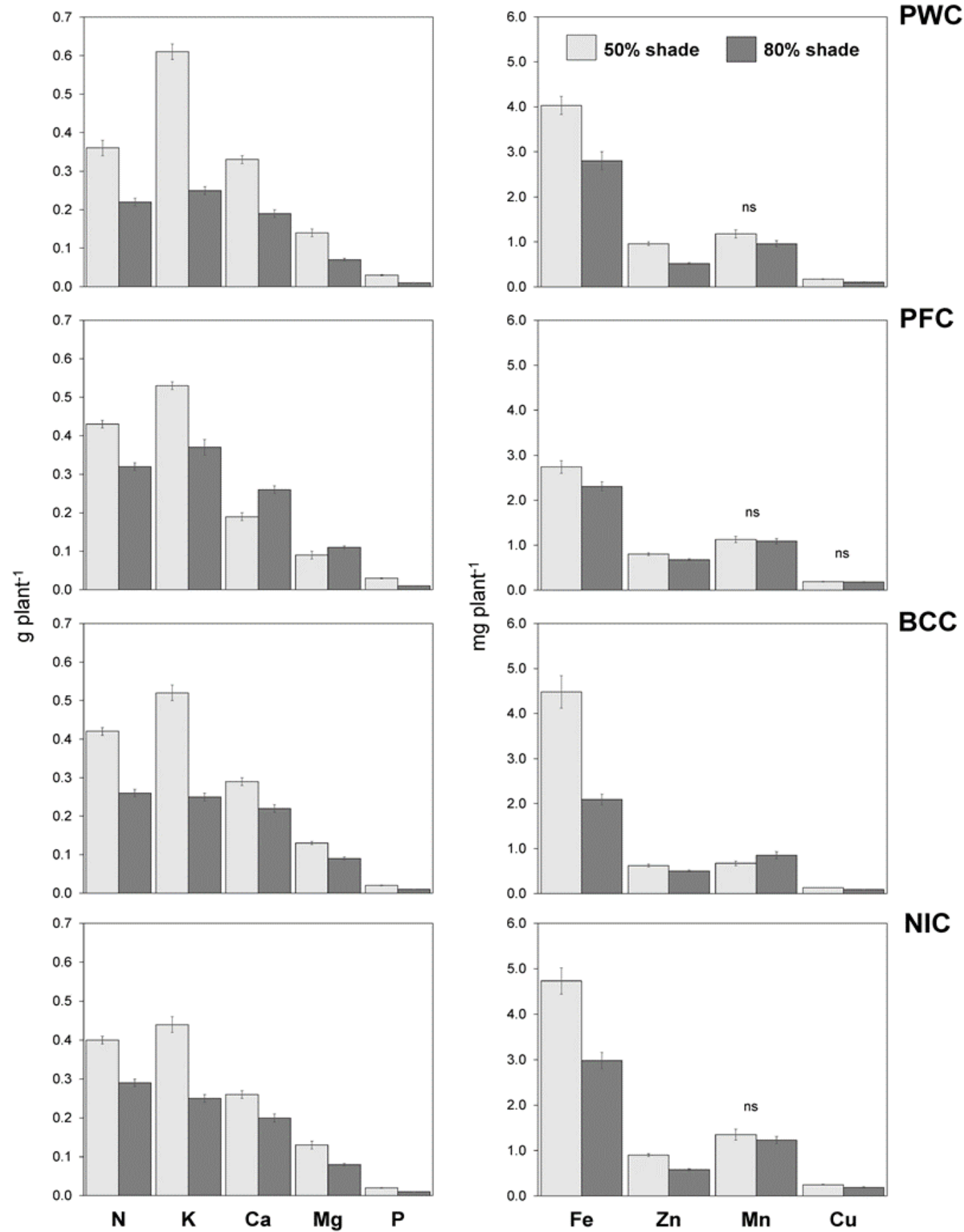

NIC

Figure 2. Nutrient uptake $\left(\mathrm{U}\right.$, Macro nutrients in $\mathrm{g} \mathrm{plant}^{-1}$ or Micro nutrients in $\mathrm{mg} \mathrm{plant}^{-1}( \pm \mathrm{SE})$ of 58 cacao genotypes subjectedto two levels of shade( $50 \%, 80 \%)$. PWC: Wild cacao (from River basins of Peruvian Amazon), PFC: Peruvian farmers' cacao, BCC: Brazilian cacao NIC: National and International cacao collections. (ns) denote not significant differences between shade levels (Scott \& Knott test, $P \leq 0.05$ ) 

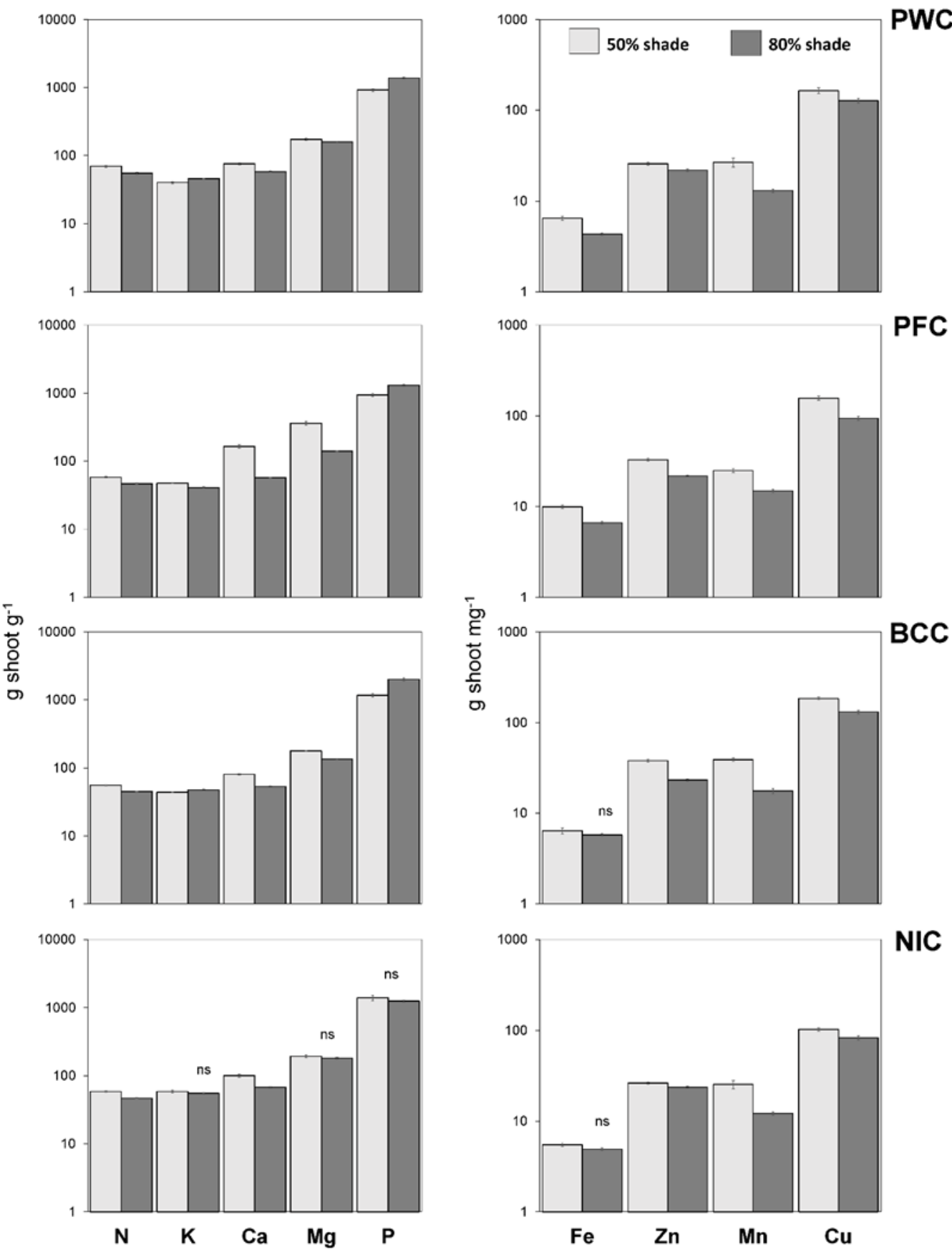

Figure 3. Nutrient uptake efficiency (NUE, Macro nutrients in g shoot $\mathrm{g}^{-1}$ or Micro nutrients in g shoot $\left.\mathrm{mg}^{-1}, \pm \mathrm{SE}\right) 58$ cacao genotypes subjected to two levels of shade $(40 \%, 80 \%)$. PWC: Wild cacao (from River basins of Peruvian Amazon), PFC: Peruvian farmers' cacao, BCC: Brazilian cacao NIC: National and International cacao collections. (ns) denote not significant differences between shade levels (Scott \& Knott test, $P \leq 0.05)$ 
Our values for $U$ were higher than those reported by Baligar et al. [43], and higher at $1000 \pm 50(50 \%$ shade $)$ than at $400 \pm 50 \mu \mathrm{mol} \mathrm{m}^{-2} \mathrm{~s}^{-1}(80 \%$ shade). These differences could be due to the fact that in the current study plants were grown a longer period (180 days vs 57 days) and with a wide collection of cacao genotypes.

Significant differences were observed in NUE of macronutrients and micronutrients between the shade levels and within each cacao collection (Figure 3). With the exception of NUE for $\mathrm{P}$ in PWC, PFC and $\mathrm{BCC}$ and $\mathrm{K}$ in $\mathrm{PWC}$ and $\mathrm{BCC}$, overall increasing shade from $50 \%$ to $80 \%$ reduced NUE for all the nutrients. However, Baligar et al. [43], reported that at ambient $\mathrm{CO}_{2}\left(380 \mu \mathrm{mol} \mathrm{mol}{ }^{-1}\right)$ increasing PPFD from 65 to $1050 \mu \mathrm{mol} \mathrm{m} \mathrm{m}^{-2} \mathrm{~s}^{-1}$ decreased the NUE of all macronutrients and micronutrients, with the exception of NUE for $\mathrm{N}$ and $\mathrm{Ca}$ which increased. In recent study, Baligar et al. [44] showed that increasing PPFD from 100 to $400 \mu \mathrm{mol} \mathrm{m} \mathrm{m}^{-2} \mathrm{~s}^{-1}$ increased uptake and NUE for all macro and micronutrients in seven cacao genotypes.

\subsection{Cacao genotypes tolerant to shade}

Shade tolerance is a complex property of plants that is achieved by different sets of responses, such as alterations in leaf physiology and biochemistry, leaf anatomy and morphology and/or plant architecture [4]. Many methods have been proposed to measure the degree of shade tolerance of several plant species such as sapling ratios (number of saplings growing in a low-light environment over the total abundance of the species), abundance-based index (number of stems, leaf density), mortality rate and relative growth (which is assumed to be larger in shade tolerant species) [1,2]. The differences observed in shade tolerance is mostly related to variations among species in adaptation of the photosynthetic apparatus to low light intensity. The effective growth of plants at low irradiance requires capacity to efficiently catch the available light and convert it into chemical energy, maintain a low rate of respiration, and use a large fraction of the carbohydrate pool for leaf growth $[38,57]$. On the other hand, shade-intolerant species tend to respond to high light regimes with much increased photosynthetic capacity, reduced leaf expansion, decreased branching and early flowering. These responses are known as shade avoidance syndrome (SAS), which is one best-studied forms of plant phenotypic plasticity [4, 76]. (Bongers et al., 2014; Martínez-García et al., 2010)

In our study significant differences were observed across cacao genotypes regarding STI as well as a moderate variability within replicates. This index varied from $25.72 \%$ to 76.15\% for AYP-22 (PWC) and ICS-1 (NIC) genotype, respectively (Table 3, Figure 4). The genotypes sensitive to shade were: AYP-22, PAS-91, PAS-105 and UGU-112 from PWC; ICT-1026 and ICT-2653 from PFC; PH-09, IPIRANGA-1, CP-53-C10, CP-49-C10 
and CEPEC-2002 from BCC; TSH-565 from NIC (Figure 4). By contrast, cacao genotypes tolerant to shade were: UGU-130 and UNG-77 from PWC; ICT-2171, ICT1087, ICT-1506 ICT-2172, ICT-2142 and ICT-2173 from PFC; PH-144, PH-990, CA-14 and PH-21 from BCC; POUND-12, UF-613, ICS-39 and ICS-1, from NIC. The rest of the genotypes were classified as medium-tolerant to shade (Figure 4).
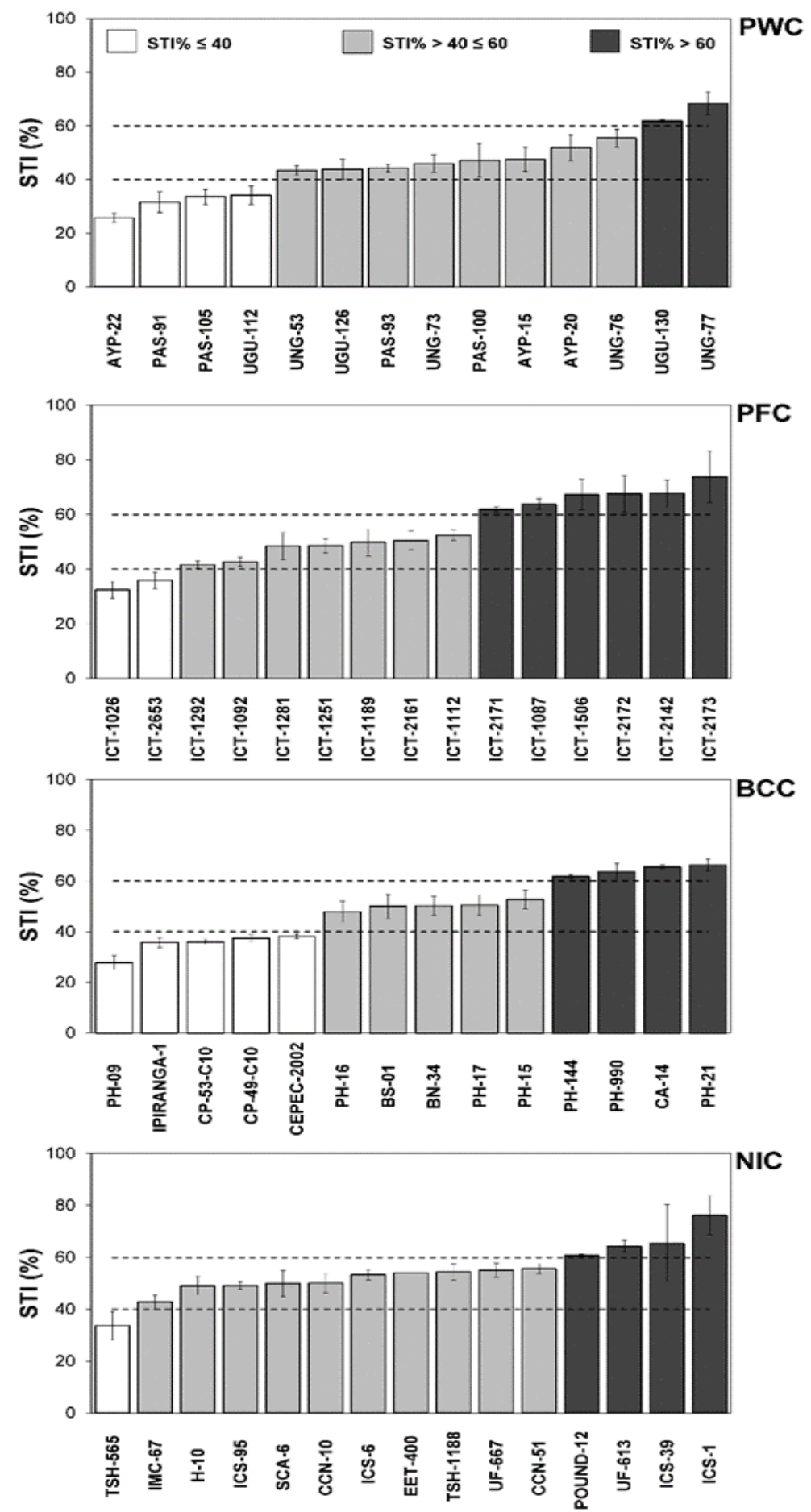

Figure 4. Shade tolerance index (STI, $\pm S D$ ) of 58 cacao genotypes subjected to two levels of shade $(40 \%, 80 \%)$. PWC: Wild cacao (from River basins of Peruvian Amazon), PFC: Peruvian farmers' cacao, BCC: Brazilian cacao NIC: National and International cacao collections. Genotypes were classified in 3 groups: sensitive to shade (STI \% $\leq 40)$, medium shade tolerant (STI \% $>40$ but $\leq 60)$ and tolerant to shade (STI \% > 60). 


\subsection{Interaction between growth, physiological parameters and nutrient uptake}

The results of the general PCA for the growth, physiological and nutrient uptake variables under two levels of shade with sensitive and tolerant cacao genotypes are shown in Figure 5. The first two PCA axis explained $51.9 \%$ of the overall variation from genotypes under $50 \%$ shade (Figure $5 \mathrm{~A}$ ), while under $80 \%$ shade the variability explained was $69.2 \%$ (Figure 5B)

At $50 \%$ shade, the first PCA axis accounted for $30.3 \%$ of overall variation and was related in negative values with high values of $\mathrm{S} / \mathrm{R}, \mathrm{CHO}, \mathrm{Ca} \mathrm{Mg}$ and $\mathrm{Fe}$ that were positively correlated among themselves and negatively correlated with $\mathrm{SL}, \mathrm{SD}, \mathrm{RDW}$ and $\mathrm{Cu}$; the sensitive genotypes PAS-105, CP-53-C10, $\mathrm{PH}-09$, CP-49-C10 were related with $\mathrm{Ca}, \mathrm{Mg}$ and Fe uptake. At the positive values were genotypes with high values of BDW, SD, $\mathrm{RDW}, \mathrm{WUE}, \mathrm{N}, \mathrm{K}, \mathrm{Mn}, \mathrm{P}$ and $\mathrm{Cu}$, that were also positively correlated among themselves. $33 \%$ and $50 \%$ of sensitive and tolerant genotypes respectively were related with $\mathrm{K}, \mathrm{Mn}$, $\mathrm{N}, \mathrm{Zn}, \mathrm{P}$ and $\mathrm{Cu}$. The second principal component, which explained $21.6 \%$ of the overall variance, was represented mainly by variations in negative values of growth parameters (RDW, SD, SL) and Cu uptake and in positive values with the rest of the nutrient and physiological parameters. The axis divides $50 \%$ of sensitive and tolerant genotypes respectively.

At $80 \%$ shade, the first PCA axis accounted for $58.4 \%$ of the overall variation and was related in negative values with $\mathrm{CHO}$ and S/R and divides $100 \%$ and $25 \%$ of sensitive and tolerant genotypes respectively. In positive values we found all shade tolerant genotypes related with physiological parameters and nutrient uptake.

Based on these results, we can infer that the sensitive and tolerant genotypes have a better development at $50 \%$ shade, while at $80 \%$ shade the tolerant genotypes are related directely with nutrient uptake, physiology and growth parameters. This characteristic of shade tolerant genotypes permits better development of plants and selection of genotypes that withstand the high level of shade, while the sensitive genotypes do not have a positive relationship with the nutrient uptake, physiology and growth parameters. 

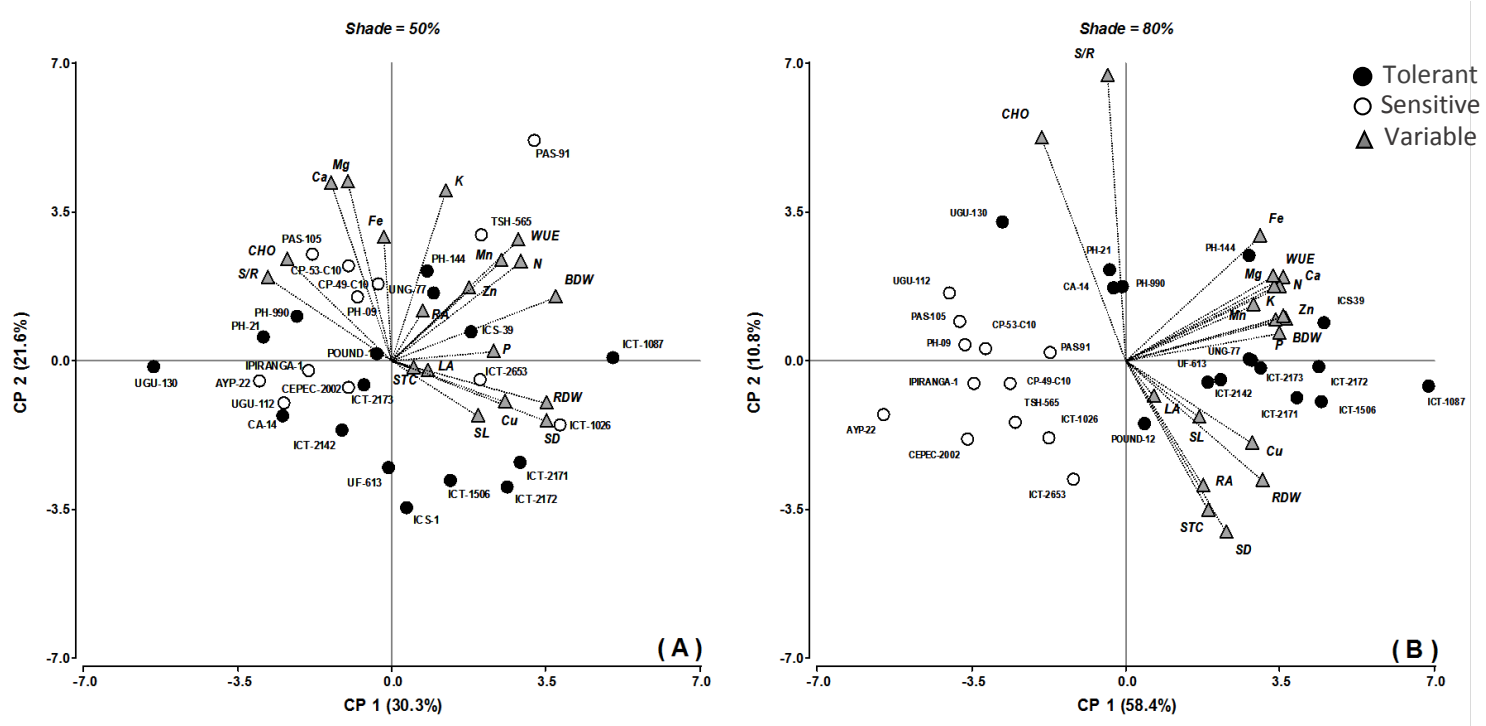

Figure 5. PCA analysis of growth, physiological parameters and nutrient uptake of shade sensitive and tolerant genotypes subjected to $50 \%(A)$ and $80 \%(B)$ shade

Usually shade intolerant species exhibit a greater physiological plasticity which allows them to achieve rapid growth rates, probably associated with more effective net assimilation rates than with structural traits $[38,77]$ The increase of light intensity when photosynthesis and growth rate are faster causes a decrease in total $\mathrm{N}$ in the leaf (source for proteins, chlorophyll, etc.) and also a decrease of $P$, that would imply that $P$ uptake cannot keep up with increased growth at higher light levels [57].

It would be expected that in cacao genotypes sensitive to shade, growth parameters have strong correlation with almost all nutrients, which is the case for RDW in tolerant genotypes. On the other hand, only shade sensitive cacao genotypes showed a positive correlation between RDW and $\mathrm{N}$ content in the aboveground tissues, probably because a long and branched root system is necessary to increase water and nutrient capture [78], that could be reduced under heavy shade conditions.

Under controlled conditions, some plants grown hydroponically have shown a decline in chlorophyll content as the Mn concentration increased [79,80], whereas in other cases it decreases as the Mn content decreased [81. In the case of cacao, all genotypes showed a negative but not significant correlation between these two parameters.

Finally, shade sensitive and tolerant cacao genotypes exhibit negative correlations between $\mathrm{Cu}$ and chlorophyll content. Several authors observed that increasing levels of $\mathrm{Cu}$ in the nutrient solution or in soil were associated to a decrease of the stomatal conductance, which causes a decline in photosynthetic gas exchange $[82,83,84]$ or that an increase of $\mathrm{Cu}$ lowered the leaf chlorophyll concentration, enhancing sensitivity to photoinhibition [85]. 


\section{Conclusions}

Fifty eight cacao genotypes grown under greenhouse conditions and subjected to $50 \%$ shade (PPFD of $1000 \pm 50 \mu \mathrm{mol} \mathrm{m}^{-2} \mathrm{~s}^{-1}$ ) and $80 \%$ shade (PPFD $400 \pm 50 \mu \mathrm{mol} \mathrm{m}^{-2} \mathrm{~s}^{-1}$ ) were evaluated to determine their growth, physiological, nutrient use efficiency responses and tolerance to shade. For almost all growth, physiological and nutritional parameters interactions between shade levels and genotypes were statistically significant, therefore, it was not possible to establish significant differences for each factor (individually). Only maximum root length, leaf area, $\mathrm{N}$ concentration, $\mathrm{Cu}$ content and $\mathrm{Cu}$ efficiency seem to vary significantly between shade levels and across genotypes.

Overall results suggest that heavy shade affects cacao negatively, except for shoot length, leaf and root area, shoot/root ratio and chlorophyll content, which are usually higher at $80 \%$ than at $50 \%$ shade.

Merely $28 \%$ of the cacao genotypes evaluated were identified as tolerant to shade, from the wild cacao, Peruvian famers' (or ICT), Brazilian, National and International cacao collections, whereas $21 \%$ were sensitive to shade.

Total dry weight and WUE showed strong relation with almost all macronutrients and micronutrients content in cacao tolerant to shade. Besides, they also have a higher total dry biomass than sensitive genotypes.

Finally, the possibility of cacao genotypes adapted to unfavorable conditions such as high shade could be used in breeding programs as a strategy to breed shade tolerant cacao cultivars to maintain sustainable cacao production under agroforestry systems. 


\section{Acknowledgments}

The project 428-PNICP-PIAP-2014/Fincyt and he project NACA 58-1275-7-112F and 58-1275-2-090F of USDA-ARS-ICT partially supported the lab and greenhouse activities of this research.

We thank Dr. Andrew Daymond, School of Agriculture, Policy and Development, University of Reading. Whiteknights, Reading UK for providing the CFC/ICCO/Biodiversity Clones used in this research; also, we thank Dr. Raul Valle of CEPLAC/CEPEC (Comissao Executiva do Plano da Lavoura Cacaueira/Centro de Pesquisas de Cacau) Ilhues/Itabuna, Bahia, Brazil for providing the Brazilian clones used for this research. We thank Lucinda Vela Vargas for management for field research of ICT. Technical and logistical facilities for the field research and laboratory analytical facilities, provided by Instituto de Cultivos Tropicales [Tropical Crops Institute] ICT/CITE Cacao y Otros Cultivos Tropicales [Productive Innovation and Technology Transfer Centre (CITE) of Cacao and Other Tropical Crops] and the Universidad Nacional Autonoma de Alto Amazonas (UNAAA), Yurimaguas, Peru are greatly appreciated. We thank Marshall Elson for excellent review of this paper.

\section{Author contributions}

E.A-G implemented the original idea, planned the experimental details and carried out the research

A.F collected and compiled reported data.

F.B took the lead in writing the manuscript, organized and interpreted the data and conducted the statistical analysis.

C.O.A-H. did plant analysis and worked the correlation matrix of all the observed parameters.

J.A. assisted with planning and implementation of the experiment.

V.C.B. conceived the study and was in charge of overall direction and planning.

All authors provided critical feedback in writing process. 


\section{References}

1. Feng, J., Zhao, K., He, D., Fang, S., Lee, T., Chu, C., He, F. Comparing shade tolerance measures of woody forest species. PeerJ 6, e5736. 2018. https://doi.org/10.7717/peerj.5736

2. Lienard, J., Florescu, I., Strigul, N. An appraisal of the classic forest succession paradigm with the shade tolerance index. PLoS ONE 10, 2015. e0117138. https://doi.org/10.1371/journal.pone.0117138

3. Valladares, F., Laanisto, L., Niinemets, Ü., Zavala, M.A. 2016. Shedding light on shade: ecological perspectives of understorey plant life. Plant Ecology \& Diversity 9 237-251. 2016. https://doi.org/10.1080/17550874.2016.1210262

4. Martínez-García, J.F., Galstyan, A., Salla-Martret, M., Cifuentes-Esquivel, N., Gallemí, M., Bou-Torrent, J. 2010. Regulatory components of shade avoidance syndrome, in: Advances in Botanical Research. Elsevier, pp. 65-116. 2010. https://doi.org/10.1016/S0065-2296(10)53003-9

5. Semchenko, M., Lepik, M., Götzenberger, L., Zobel, K. Positive effect of shade on plant growth: amelioration of stress or active regulation of growth rate?: Effects of shade on plant growth. Journal of Ecology 100, 459-466. 2012. https://doi.org/10.1111/j.1365-2745.2011.01936.x

6. Lahive, F., Hadley, P., Daymond, A.J. The physiological responses of cacao to the environment and the implications for climate change resilience. A review. Agronomy for Sustainable Development 39, 5. 2019. https://doi.org/10.1007/s13593-018-0552-0

7. De Almeida, A.-A.F. de, Valle, R.R. Ecophysiology of the cacao tree. Brazilian Journal of Plant Physiology 19, 425-448. 2007. https://doi.org/10.1590/S167704202007000400011

8. Rice, R.A., Greenberg, R. Cacao Cultivation and the Conservation of Biological Diversity. AMBIO: A Journal of the Human Environment 29 167-173. 2000. https://doi.org/10.1579/0044-7447-29.3.167

9. Acheampong, K., Hadley, P., Daymond, A.J. Photosynthetic activity and early growth of four cacao genotypes as influenced by different shade regimes under West African dry and wet season conditions. Ex. Agric. 49, 31-42. 2013. https://doi.org/10.1017/S0014479712001007

10. Da Silva Branco, M.C., De Almeida, A.-A.F., Dalmolin, Â.C., Ahnert, D., Baligar, V.C. Influence of low light intensity and soil flooding on cacao physiology. Scientia Horticulturae 217 243-257. 2017. https://doi.org/10.1016/j.scienta.2017.01.038

11. Galyuon, I.K.A., McDavid, C.R., Lopez, F.B., Spence, J.A.. The effect of irradiance level on cocoa (Theobroma cacao L): I. Growth and leaf adaptations. Trop. Agric. 73 23-28. 1996.

12. Galyuon, I.K.A., McDavid, C.R., Lopez, F.B., Spence, J.A. The effect of irradiance level on coco (Theobroma cacao L.): II. Gas exchange and chlorophyll fluorescence. Trop. Agric. 73 29-33. 1996.

13. Jagoret, P., Ngnogue, H.T., Malézieux, E., Michel, I. Trajectories of cocoa agroforests and their drivers over time: Lessons from the Cameroonian experience. European Journal of Agronomy 101 183-192. 2018. https://doi.org/10.1016/j.eja.2018.09.007

14. Nijmeijer, A., Lauri, P.-E., Harmand, J.-M., Freschet, G.T., Essobo Nieboukaho, J.D., Fogang, P.K., Enock, S., Saj, S. Long-term dynamics of cocoa agroforestry systems established on lands previously occupied by savannah or forests. Agriculture, Ecosystems \& Environment 275 100-111. 2019. https://doi.org/10.1016/j.agee.2019.02.004

15. Saj, S., Durot, C., Mvondo Sakouma, K., Tayo Gamo, K., Avana-Tientcheu, M.-L.. Contribution of associated trees to long-term species conservation, carbon storage and sustainability: a functional analysis of tree communities in cacao plantations of 
Central Cameroon. International Journal of Agricultural Sustainability 15 282-302. 2017. https://doi.org/10.1080/14735903.2017.1311764

16. Tscharntke, T., Clough, Y., Bhagwat, S.A., Buchori, D., Faust, H., Hertel, D., Hölscher, D., Juhrbandt, J., Kessler, M., Perfecto, I., Scherber, C., Schroth, G., Veldkamp, E., Wanger, T.C. Multifunctional shade-tree management in tropical agroforestry landscapes - a review: Multifunctional shade-tree management. Journal of Applied Ecology 48, 619-629. 2011. https://doi.org/10.1111/j.13652664.2010.01939.x

17. Asante, W.A., Acheampong, E., Kyereh, E., Kyereh, B. Farmers' perspectives on climate change manifestations in smallholder cocoa farms and shifts in cropping systems in the forest-savannah transitional zone of Ghana. Land Use Policy 66, 374-381. 2017. https://doi.org/10.1016/j.landusepol.2017.05.010

18. Niether, W., Armengot, L., Andres, C., Schneider, M., Gerold, G. Shade trees and tree pruning alter through fall and microclimate in cocoa (Theobroma cacao L.) production systems. Annals of Forest Science 75, 38. 2018. https://doi.org/10.1007/s13595-018-0723-9

19. Abou Rajab, Y., Leuschner, C., Barus, H., Tjoa, A., Hertel, D. Cacao cultivation under diverse shade tree cover allows high carbon storage and sequestration without yield losses. PLoS ONE 11, e0149949. 2016. https://doi.org/10.1371/journal.pone.0149949

20. Andres, C., Blaser, W.J., Dzahini-Obiatey, H.K., Ameyaw, G.A., Domfeh, O.K., Awiagah, M.A., Gattinger, A., Schneider, M., Offei, S.K., Six, J. Agroforestry systems can mitigate the severity of cocoa swollen shoot virus disease. Agriculture, Ecosystems \& Environment 252, 83-92. 2018. https://doi.org/10.1016/j.agee.2017.09.031

21. Bos, M.M., Steffan-Dewenter, I., Tscharntke, T. Shade tree management affects fruit abortion, insect pests and pathogens of cacao. Agriculture, Ecosystems \& Environment 120 201-205. 2007. https://doi.org/10.1016/j.agee.2006.09.004

22. Bunn, C., Läderach, P., Quaye, A., Muilerman, S., Noponen, M.R.A., Lundy, M. Recommendation domains to scale out climate change adaptation in cocoa production in Ghana. Climate Services 100123. 2019. https://doi.org/10.1016/j.cliser.2019.100123

23. Hosseini Bai, S., Trueman, S.J., Nevenimo, T., Hannet, G., Bapiwai, P., Poienou, M., Wallace, H.M. Effects of shade-tree species and spacing on soil and leaf nutrient concentrations in cocoa plantations at 8 years after establishment. Agriculture, Ecosystems \& Environment 246 134-143. 2017. https://doi.org/10.1016/j.agee.2017.06.003

24. Sambuichi, R.H.R., Vidal, D.B., Piasentin, F.B., Jardim, J.G., Viana, T.G., Menezes, A.A., Mello, D.L.N., Ahnert, D., Baligar, V.C. Cabruca agroforests in southern Bahia, Brazil: tree component, management practices and tree species conservation. Biodivers Conserv 21 1055-1077. 2012. https://doi.org/10.1007/s10531-012-0240-3

25. Vanhove, W., Vanhoudt, N., Van Damme, P. Effect of shade tree planting and soil management on rehabilitation success of a 22-year-old degraded cocoa (Theobroma cacao L.) plantation. Agriculture, Ecosystems \& Environment 21914 25. 2016. https://doi.org/10.1016/j.agee.2015.12.005

26. Vliet, J. A. van, Giller, K.E. Mineral nutrition of cocoa: a review, Editor(s): Donald L. Sparks, Advances in Agronomy. Academic Press, Vol. 141, pag 185-270. 2017

27. Abdulai, I., Jassogne, L., Graefe, S., Asare, R., Van Asten, P., Läderach, P., Vaast, $P$. Characterization of cocoa production, income diversification and shade tree management along a climate gradient in Ghana. PLoS ONE 13, e0195777. 2018. https://doi.org/10.1371/journal.pone.0195777

28. Asare, R., Markussen, B., Asare, R.A., Anim-Kwapong, G., Ræbild, A. On-farm cocoa yields increase with canopy cover of shade trees in two agro-ecological 
zones in Ghana. Climate and Development 11, 435-445. 2019.

https://doi.org/10.1080/17565529.2018.1442805

29. Blaser, W.J., Oppong, J., Hart, S.P., Landolt, J., Yeboah, E., Six, J. Climate-smart sustainable agriculture in low-to-intermediate shade agroforests. Nat Sustain 1 234-239. 2018. https://doi.org/10.1038/s41893-018-0062-8

30. Blaser, W.J., Oppong, J., Yeboah, E., Six, J. Shade trees have limited benefits for soil fertility in cocoa agroforests. Agriculture, Ecosystems \& Environment 243, 8391. 2017 https://doi.org/10.1016/j.agee.2017.04.007

31. Jezeer, R.E., Verweij, P.A., Santos, M.J., Boot, R.G.A. Shaded Coffee and Cocoa Double Dividend for Biodiversity and Small-scale Farmers. Ecological Economics 140 136-145. 2017. https://doi.org/10.1016/j.ecolecon.2017.04.019

32. Suárez Salazar, J.C., Melgarejo, L.M., Casanoves, F., Di Rienzo, J.A., DaMatta, F.M., Armas, C. 2018. Photosynthesis limitations in cacao leaves under different agroforestry systems in the Colombian Amazon. PLoS ONE 13, e0206149. 2018. https://doi.org/10.1371/journal.pone.0206149

33. Wood, G.A.R. and Lass, R.A. 2001. Cocoa, 4th edition. Blackwell Science, Oxford, UK.619 p.

34. Chazdon, R.L. Sunflecks and their importance to forest understorey plants, in: Advances in ecological research. Elsevier, pp. 1-63. 1988. https://doi.org/10.1016/S0065-2504(08)60179-8

35. De Castro, F. Light spectral composition in a tropical forest: measurements and model. Tree Physiology 20, 49-56. 2000. https://doi.org/10.1093/treephys/20.1.49

36. Goldstein, G., Santiago, L.S., Campanello, P.I., Avalos, G., Zhang, Y.-J., Villagra, M. Facing shortage or excessive light: how tropical and subtropical trees adjust their photosynthetic behavior and life history traits to a dynamic forest environment, in: Goldstein, G., Santiago, L.S. (Eds.), Tropical tree physiology. Springer International Publishing, Cham, pp. 319-336. 2016. https://doi.org/10.1007/978-3319-27422-5_15

37. Liang, N., Tang, Y., Okuda, T. 2001. Is elevation of carbon dioxide concentration beneficial to seedling photosynthesis in the understory of tropical rain forests? Tree Physiology 21 1047-1055. 2001. https://doi.org/10.1093/treephys/21.14.1047

38. Pallardy, S.G. Photosynthesis, in: Physiology of woody plants. Elsevier, pp. 107167. 2008. https://doi.org/10.1016/B978-012088765-1.50006-3

39. Pearcy, R.W. Photosynthetic gas exchange responses of Australian tropical forest trees in canopy, gap and understory micro-environments. Functional Ecology 1 169. 1987. https://doi.org/10.2307/2389419

40. Tang, Y., Kachi, N., Furukawa, A., Awang, M.B. Heterogeneity of light availability and its effects on simulated carbon gain of tree leaves in a small gap and the understory in a tropical rain forest. Biotropica 31 268-278. 1999.

https://doi.org/10.1111/j.1744-7429.1999.tb00138.x

41. Miyaji, K.-I., Da Silva, W.S., Alvim, P.D.T. Longevity of leaves of a tropical tree, Theobroma cacao, grown under shading, in relation to position within the canopy and time of emergence. New Phytol 135, 445-454. 2008.

https://doi.org/10.1046/j.1469-8137.1997.00667.x

42. Baligar, V.C., Bunce, J.A., Machado, R.C.R., Elson, M.K. Photosynthetic photon flux density, carbon dioxide concentration, and vapor pressure deficit effects on photosynthesis in cacao seedlings. Photosynthetica 46 216-221. 2008. https://doi.org/10.1007/s11099-008-0035-7

43. Baligar, V.C., Bunce, J.A., Machado, R.C.R., Elson, M.K. Carbon dioxide and photosynthetic photon flux density effects on growth and mineral uptake of cacao. J. Food Agric. Environ. 3 142-147. 2005.

44. Baligar, V.C.; Elson, M.K.; Almeida, A.A.F.; de Araujo, Q.R.; Ahnert, D.; He, Z. The impact of carbon dioxide concentrations and low to adequate photosynthetic photon flux density on growth, physiology and nutrient use efficiency of juvenile 
cacao genotypes. Agronomy 2021, 11, 397.

https://doi.org/10.3390/agronomy11020397

45. Bastid, P., Jimmy, I. Gas transfer measurements on young cocoa trees in field and modeling of photosynthetic activity. Presented at the 14th International Cocoa Research Conference, Accra, Ghana, pp. 195-203. 2003.

46. Hutcheon, W.F. Photosynthesis of cocoa: Photosynthesis in relation to the light and plant nutrient status. 1973-74. Cocoa Research Institute of Ghana. 1976.

47. Raja Harun, R.M., Hardwick, K. The effects of prolonged exposure to different light intensities on the photosynthesis of cocoa leaves. Presented at the 10th International Cocoa Research Conference, Santo Domingo, Dominican Republic, pp. 205-209. 1988.

48. Daymond, A.J., Hadley, P., Machado, R.C.R., Ng, E. Canopy characteristics of contrasting clones of cacao (Theobroma cacao). Experimental Agriculture 38, 359367. 2002. https://doi.org/10.1017/S0014479702003083

49. Motamayor, J.C., Lachenaud, P., da Silva e Mota, J.W., Loor, R., Kuhn, D.N., Brown, J.S., Schnell, R.J. 2008. Geographic and genetic population differentiation of the Amazonian chocolate tree (Theobroma cacao L). PLoS ONE 3, e3311. 2008. https://doi.org/10.1371/journal.pone.0003311

50. Pereira de Araújo, R., Furtado de Almeida, A.-A., Pinto Barroso, J., Aparecida de Oliveira, R., Pinto Gomes, F., Ahnert, D., Baligar, V. Molecular and morphophysiological responses cocoa leaves with different concentrations of anthocyanin to variations in light levels. Scientia Horticulturae 224 188-197. 2017. https://doi.org/10.1016/j.scienta.2017.06.008

51. Silva, F.C. Manual de análises químicas de solos, plantas e fertilizantes. $2^{\mathrm{a}}$ edição revista e ampliada. Embrapa Informação Tecnológica Brasília, DF. 627p. 2009.

52. Lamari, L. ASSESS 2.0. Image Analysis Sofware for Plant Disease Quantification. American Phytopathological Society. APS press. 125p. 2008.

53. United States Environmental Protection Agency (US EPA). Method 3050B: Acid Digestion of Sediments, Sludges, and Soils, Revision 2. Washington, DC. 1996.

54. Baligar, V.C., Fageria, N.K., He, Z.L.. Nutrient use efficiency in plants. Communications in Soil Science and Plant Analysis 32, 921-950. 2001. https://doi.org/10.1081/CSS-100104098

55. McDonald, G., Bovill, W., Huang, C., Lightfoot, D. Nutrient use efficiency, in: Kole, C. (Ed.), Genomics and breeding for climate-resilient crops. Springer Berlin Heidelberg, Berlin, Heidelberg, pp. 333-393. 2013. https://doi.org/10.1007/978-3642-37048-9_10

56. Di Rienzo J.A., Casanoves F., Balzarini M.G., Gonzalez L., Tablada M., Robledo C.W. InfoStat versión 2020. Centro de Transferencia InfoStat, FCA, Universidad Nacional de Córdoba, Argentina. 2020. URL http://www.infostat.com.ar

57. Poorter, H., Niklas, K.J., Reich, P.B., Oleksyn, J., Poot, P., Mommer, L. Biomass allocation to leaves, stems and roots: meta-analyses of interspecific variation and environmental control: Tansley review. New Phytologist 193, 30-50. 2012. https://doi.org/10.1111/j.1469-8137.2011.03952.x

58. Wartenberg, A.C., Blaser, W.J., Roshetko, J.M. et al. Soil fertility and Theobroma cacao growth and productivity under commonly intercropped shade-tree species in Sulawesi, Indonesia. Plant Soil 453, 87-104. 2020. https://doi.org/10.1007/s11104018-03921-x

59. Daymond, A.J., Hadley, P. The effects of temperature and light integral on early vegetative growth and chlorophyll fluorescence of four contrasting genotypes of cacao (Theobroma cacao). Ann Applied Biology 145 257-262. 2004. https://doi.org/10.1111/j.1744-7348.2004.tb00381.x

60. Daymond, A.J., Tricker, P.J., Hadley, P. Genotypic variation in photosynthesis in cacao is correlated with stomatal conductance and leaf nitrogen. Biologia Plantarum 55, 99-104. 2011. https://doi.org/10.1007/s10535-011-0013-y 
61. Niether, W., Schneidewind, U., Fuchs, M., Schneider, M., Armengot, L. Below- and aboveground production in cocoa monocultures and agroforestry systems. Science of The Total Environment 657, 558-567. 2019.

https://doi.org/10.1016/j.scitotenv.2018.12.050

62. De Almeida, A.-A.F., Valle, R.R. Cacao: ecophysiology of growth and production, in: DaMatta, F. (Ed.), Ecophysiology of tropical tree crops. Nova Science Publishers, New York, p. 389. 2010.

63. Acheampong, K., Hadley, P., Daymond, A., Adu-Yeboah, P. The influence of shade and organic fertilizer treatments on the physiology and establishment of Theobroma cacao clones. AJEA 6, 347-360. 2015. https://doi.org/10.9734/AJEA/2015/15206

64. Agudelo-Castañeda, G.A., Cadena-Torres, J., Almanza-Merchán, P.J., PinzónSandoval, E.H. Desempeño fisiológico de nueve genotipos de cacao (Theobroma cacao L.) bajo la sombra de tres especies forestales en Santander, Colombia. Rev. Colomb. Cienc. Hortic. 12 223-232. 2018. https://doi.org/10.17584/rcch.2018v12i1.7341

65. Jaimez, R.E., Amores Puyutaxi, F., Vasco, A., Loor, R.G., Tarqui, O., Quijano, G., Jimenez, J.C., Tezara, W. Photosynthetic response to low and high light of cacao growing without shade in an area of low evaporative demand. Acta Biológica Colombiana 23, 95-103. 2018. https://doi.org/10.15446/abc.v23n1.64962

66. López-Marín, J., Gálvez, A., González, A., Egea-Gilabert, C., Fernández, J.A. Effect of shade on yield, quality and photosynthesis-related parameters of sweet pepper plants. Acta Horticulturae, (956), 545-552. 2012.

67. Yang M., Liu M., Lu J., Yang H. Effects of shading on the growth and leaf photosynthetic characteristics of three forages in an apple orchard on the Loess Plateau of eastern Gansu, China. PeerJ 7:e7594. 2019. https://doi.org/10.7717/peerj.7594

68. Baliza, D. P., Cunha, R.L., Guimarães, R.J., Barbosa, J.P.R.A.D., Ávila, F W., Passos, A.M.A. Physiological characteristics and development of coffee plants under different shading levels. Rev. Bras. Ciênc. Agrár. Recife, 7: p.37-43. 2012. https://doi.org/10.5039/agraria.v7i1a1305

69. Cabala Rosand, P., Santana, M.B.M., de Santana, C.J.L. Cacao, in: Plucknett, D., Sprague, H.B. (Eds.), Detecting mineral nutrient deficiencies in tropical and temperate crops. CRC Press. 1989. https://doi.org/10.1201/9780429035258

70. Murray DB. Leaf analysis applied to cocoa. Cocoa Growers' Bulletin. 9:25-35. 1967.

71. Ahenkorah, Y., Halm, B., Appiah, M., Akrofi, G., \& Yirenkyi, J. Twenty Years' Results from a Shade and Fertilizer Trial on Amazon Cocoa (Theobroma cacao) in Ghana. Experimental Agriculture, 23(1), 31-39. 1987. doi:10.1017/S0014479700001101

72. Loue, A. Etude des carences et des deficincies minerales sur le cacaoyer (Study of deficiencies and mineral deficiencies of cacao). IFCC Bulletin 1:53. 1961

73. Snoeck, J. Cacao. In: Martin-Prevel, P., Gagnard, J. and Gautier, P. (ed) L'analyse végétale dans le contrôle de l'alimentation des plantes temperées et tropicales. Technique et doc. (Plant Analysis of Nutrient Requirements in Temperate and Tropical Plants). Lavoisier Publishing Inc., NY. pp. 432-439. 1987.

74. Bhargava, B.S. and Raghupati, H.B. Analysis of plants. In: Tondon, H.S. (ed) Methods of Analysis of Soils, Water and Fertilizers. FDCO, New Delhi, pp. 49-82. 1993.

75. Isaac, M.E., Timmer, V.R., Quashie-Sam, S.J. Shade tree effects in an 8-year-old cocoa agroforestry system: biomass and nutrient diagnosis of Theobroma cacao by vector analysis. Nutrient Cycling in Agroecosystems 78 155-165. 2007. https://doi.org/10.1007/s10705-006-9081-3

76. Bongers, F.J., Evers, J.B., Anten, N.P.R., Pierik, R. From shade avoidance responses to plant performance at vegetation level: using virtual plant modelling as a tool. New Phytol 204 268-272. 2014. https://doi.org/10.1111/nph.13041 
77. Portsmuth, A., Niinemets, Ü. Structural and physiological plasticity in response to light and nutrients in five temperate deciduous woody species of contrasting shade tolerance. Funct Ecology 21. 2007. https://doi.org/10.1111/j.13652435.2006.01208.x

78. Markesteijn, L., Poorter, L. Seedling root morphology and biomass allocation of 62 tropical tree species in relation to drought- and shade-tolerance. Journal of Ecology 97, 311-325. 2009. https://doi.org/10.1111/j.1365-2745.2008.01466.x

79. Macfie, S.M., Taylor, G.J. The effects of excess manganese on photosynthetic rate and concentration of chlorophyll in Triticum aestivum grown in solution culture. Physiol Plant 85, 467-475. 1992. https://doi.org/10.1111/j.13993054.1992.tb05813.x

80. Moroni, J.S., Briggs, K.G., Taylor, G.J. Chlorophyll content and leaf elongation rate in wheat seedlings as a measure of manganese tolerance. Plant Soil 136 1-9. 1991. https://doi.org/10.1007/BF02465214

81. Singh, P., Misra, A., Srivastava, N.K. Influence of Mn deficiency on growth, chlorophyll content, physiology, and essential monoterpene oil(s) in genotypes of spearmint (Mentha spicata L.). Photosynthetica 39, 473-476. 2001. https://doi.org/10.1023/A:1015107116205

82. Bazihizina, N., Colzi, I., Giorni, E., Mancuso, S., Gonnelli, C. Photosynthesizing on metal excess: Copper differently induced changes in various photosynthetic parameters in copper tolerant and sensitive Silene paradoxa L. populations. Plant Science 232, 67-76. 2015. https://doi.org/10.1016/j.plantsci.2014.12.015

83. Gonzalez-Mendoza, D., Gil, F.E. y, Escoboza-Garcia, F., Santamaria, J.M., ZapataPerez, O. Copper stress on photosynthesis of black mangle (Avicennia germinans). An. Acad. Bras. Ciênc. 85, 665-670. 2013. https://doi.org/10.1590/S0001-37652013000200013

84. Moustakas, M., Ouzounidou, G., Symeonidis, L., Karataglis, S. Field study of the effects of excess copper on wheat photosynthesis and productivity. Soil Science and Plant Nutrition 43, 531-539. 1997. https://doi.org/10.1080/00380768.1997.10414780

85. Pätsikkä, E., Kairavuo, M., Šeršen, F., Aro, E.-M., Tyystjärvi, E. Excess copper predisposes photosystem II to photoinhibition in vivo by outcompeting iron and causing decrease in leaf chlorophyll. Plant Physioly 129 1359-1367. 2002. https://doi.org/10.1104/pp.004788 